\title{
Treatment of Patients with Early Breast Cancer: Evidence, Controversies, Consensus
}

German Expert Opinions on the 17th International St. Gallen Consensus Conference

\section{Behandlung von Patientinnen mit frühem Mammakarzinom: Evidenz, Kontroversen, Konsens}

\author{
Meinungsbild deutscher Expert* Innen zur 17. Internationalen \\ St.-Gallen-Konsensuskonferenz
}

\section{(우 (1) (요 $\odot$}

Authors

Michael Untch ${ }^{1 *}$, Peter A. Fasching ${ }^{2 *}$, Sara Y. Brucker ${ }^{3 * * \#}$, Wilfried Budach ${ }^{* *}$, Carsten Denkert ${ }^{5 * * \#}$,

Renate Haidinger ${ }^{*}$, Jens Huober ${ }^{7,8 * \#}$, Christian Jackisch ${ }^{9 *}$, Wolfgang Janni ${ }^{10 *}$, Cornelia Kolberg-Liedtke ${ }^{11,12,13 *}$,

David Krug ${ }^{14 *}$, Thorsten Kühn ${ }^{15 *}$, Sibylle Loibl ${ }^{16,17 * * \#, ~ D i a n a ~ L u ̈ f t n e r ~}{ }^{18 *}$, Volkmar Müller ${ }^{19 * *}$,

Andreas Schneeweiss ${ }^{20,21 *}$, Marc Thill ${ }^{22 *}$, Nadia Harbeck ${ }^{23 * \#}$, Christoph Thomssen ${ }^{24 *}$

Affiliations

1 Klinik für Gynäkologie und Geburtshilfe, interdisziplinäres Brustzentrum, HELIOS Klinikum Berlin Buch, Berlin, Germany

2 Frauenklinik des Universitätsklinikums Erlangen, Comprehensive Cancer Center Erlangen-EMN, Friedrich-AlexanderUniversität Erlangen-Nürnberg, Erlangen, Germany

3 Universitäts-Frauenklinik Tübingen, Tübingen, Germany

4 Klinik für Strahlentherapie und Radioonkologie, Universitätsklinik Düsseldorf, Düsseldorf, Germany

5 Pathologisches Institut, Philipps Universität Marburg und Universitätsklinikum Marburg (UKGM), Marburg, Germany

6 Brustkrebs Deutschland e.V., Hohenbrunn, Germany

7 Brustzentrum Kantonsspital St. Gallen, St. Gallen, Switzerland

8 Brustzentrum, Universitätsfrauenklinik Ulm, Ulm, Germany 9 Klinik für Gynäkologie und Geburtshilfe, Sana-Klinikum Offenbach GmbH, Offenbach, Germany

10 Universitätsfrauenklinik Ulm, Ulm, Germany

11 Klinik für Frauenheilkunde und Geburtshilfe, Universitätsklinikum Essen, Essen, Germany

12 palleos healthcare $\mathrm{GmbH}$, Wiesbaden, Germany

13 Phaon scientific GmbH, Wiesbaden, Germany

14 Klinik für Strahlentherapie (Radioonkologie), Universitätsklinikum Schleswig-Holstein, Campus Kiel, Kiel, Germany

15 Klinik für Frauenheilkunde und Geburtshilfe, Klinikum Esslingen, Esslingen, Germany

16 German Breast Group (GBG), Neu-Isenburg, Germany
17 Centrum für Hämatologie und Onkologie Bethanien, Frankfurt am Main, Germany

18 Medizinische Klinik mit Schwerpunkt Hämatologie, Onkologie und Tumorimmunologie, Charité Campus VirchowKlinikum, Berlin, Germany

19 Klinik und Poliklinik für Gynäkologie, Universitätsklinik Hamburg-Eppendorf (UKE), Hamburg, Germany

20 Sektionsleiter Gynäkologische Onkologie, Nationales Centrum für Tumorerkrankungen (NCT) Universitätsklinikum Heidelberg, Heidelberg, Germany

21 Deutsches Krebsforschungszentrum (DKFZ), Heidelberg, Germany

22 Klinik für Gynäkologie und Gynäkologische Onkologie, Interdisziplinäres Brustzentrum, Agaplesion Markus Krankenhaus, Frankfurt am Main, Germany

23 Brustzentrum, Frauenklinik, LMU Klinikum, München, Germany

24 Universitätsklinik und Poliklinik für Gynäkologie, MartinLuther-Universität Halle-Wittenberg, Halle (Saale), Germany

Key words

St. Gallen Consensus 2021, early breast cancer, surgery, radiotherapy, (neo)adjuvant systemic therapy, targeted therapy

\section{Schlüsselwörter}

St.-Gallen-Konsensus 2021, frühes Mammakarzinom, Operation, Strahlentherapie, (neo)adjuvante Systemtherapie, zielgerichtete Therapie received

accepted after revision

published online
8. 4.2021

20.4. 2021

19.5. 2021 
Bibliography

Geburtsh Frauenheilk 2021; 81: 637-653

DOI 10.1055/a-1483-2782

ISSN 0016-5751

(C) 2021. The Author(s).

This is an open access article published by Thieme under the terms of the Creative Commons Attribution-NonDerivative-NonCommercial-License, permitting copying and reproduction so long as the original work is given appropriate credit. Contents may not be used for commercial purposes, or adapted, remixed, transformed or built upon. (https://creativecommons.org/licenses/by-nc-nd/4.0/)

Georg Thieme Verlag KG, Rüdigerstraße 14,

70469 Stuttgart, Germany

Correspondence

Prof. Dr. med. Michael Untch

Klinik für Gynäkologie und Geburtshilfe, interdisziplinäres

Brustzentrum, HELIOS Klinikum Berlin Buch

Schwanebecker Chaussee 50, 13125 Berlin, Germany

michael.untch@helios-gesundheit.de

$\circledast$

Deutsche Version unter:

https://doi.org/10.1055/a-1483-2782

\begin{abstract}
This year's 17th St. Gallen (SG) Consensus Conference on the Treatment of Patients with Early Breast Cancer (SG-BCC) with the title "Customizing local and systemic therapies for women with early breast cancer" focused on the challenge of targeting the treatment of early breast cancer more specifically to the individual disease situation of each patient. As in previous years, a German working group of leading breast cancer experts discussed the results of the international SG-BCC 2021 in the context of the German guideline. It is helpful to compare the SG recommendations with the recently updated treatment recommendations of the Breast Commission of
\end{abstract}

the German Working Group on Gynaecological Oncology (Arbeitsgemeinschaft Gynäkologische Onkologie e. V., AGO) and the $\mathrm{S} 3$ guideline because the SG-BCC panel comprised experts from different countries, which is why country-specific aspects can be incorporated into the SG recommendations. The German treatment recommendations of the AGO and the $\mathrm{S} 3$ guideline are based on current evidence. Nevertheless, any therapeutic decision must always undergo a risk-benefit analysis for the specific situation and to be discussed with the patient.

\section{ZUSAMMENFASSUNG}

Die diesjährige 17. St.-Gallen-(SG-)Konsensus-Konferenz zur Behandlung von Patientinnen mit frühem Mammakarzinom (SG-BCC) stand unter dem Motto „Customizing local and systemic therapies for women with early breast cancer" und fokussierte auf die Herausforderung, die Behandlung des frühen Mammakarzinoms zielgerichteter auf die individuelle Krankheitssituation adaptieren. Wie schon in den vergangenen Jahren hat auch dieses Jahr eine deutsche Arbeitsgruppe führender Brustkrebsexpert* Innen die Ergebnisse der internationalen SG-BCC 2021 vor dem Hintergrund der deutschen Therapieempfehlungen für den Klinikalltag in Deutschland diskutiert. Der Vergleich der SG-Empfehlungen mit den erst kürzlich aktualisierten Therapieempfehlungen der Kommission Mamma der Arbeitsgemeinschaft Gynäkologische Onkologie e. V. (AGO) sowie der S3-Leitlinie ist hilfreich, da sich das SG-BCC-Panel aus Experten unterschiedlicher Länder zusammensetzt, weshalb länderspezifische Besonderheiten in die SG-Empfehlungen einfließen können. Die deutschen Therapieempfehlungen der AGO sowie der S3-Leitlinie basieren auf der aktuellen Evidenz. Gleichwohl muss jede Therapieentscheidung immer einer Nutzen-Risiko-Abwägung für die individuelle Situation unterzogen und mit der Patientin besprochen werden.

\section{Introduction}

The motto of this year's 17th St. Gallen (SG) Conference on "Primary Treatment of Early Breast Cancer" (SG-BCC) was "Customising local and systemic therapies for women with early breast cancer". Targeting the treatment of early breast cancer more and more to the specific disease situation of each patient is a clinical challenge. The 60 breast cancer experts came from 25 countries, including five panel members from Germany (see $>$ Table 1). The SG-BCC recommendations are based on a majority vote of the panellists aiming to establish an international consensus for everyday clinical practice. The panellists come from a number of different countries with different health systems and resources. It is not surprising that this would also be reflected in the consensus. For some years now, a German working group has been commenting on the voting results of the SG-BCC panel and their agreement with the treatment recommendations of the Breast Commission of the "Arbeitsgemeinschaft Gynäkologische Onko- logie e.V." (AGO Mamma) [1], which updates its recommendations every year.

\section{Genetic Testing for High-Risk Mutations}

\section{General considerations}

Genetic testing for mutations in high-risk genes (e.g. BRCA1/2) requires appropriate patient information and counselling. The German experts agree with the majority vote $(78 \%)$ of the SG-BCC panellists that patients with a calculated risk of a pathogenic germline mutation $>10 \%$ should be offered genetic testing (Level of Evidence [LoE] 2bB AGO++). For further details, the German experts refer to the current recommendations of the AGO Mamma [1].

\section{Genetic testing of which genes?}

In addition to mutations in the known risk genes $B R C A 1 / 2$, the SG-BCC panellists $(67 \%)$ recommend mutation analysis of other 
- Table 1 International SG-BCC Panel 2021.

Chair: Eric P. Winer (USA)

Co-Chairs: Harold Burstein (USA), Giuseppe Curigliano (Italy), Michael Gnant (Austria), Meredith Regan (USA), Beat Thürlimann (Switzerland), Walter Weber (Switzerland)

- Stephan Aebi (Switzerland)

- Fabrice André (France)

- Carlos Barrios (Brazil)

- Jonas Bergh (Sweden)

- Hervé Bonnefoi (France)

- Denisse Bretel Morales (Peru)

- Sara Y. Brucker (Germany)

- Harold Burstein (USA)

- David Cameron (UK)

- Fatima Cardoso (Portugal)

- Lisa Carey (USA)

- Boon Chua (Australia)

- Eva Ciruelos (Spain)

- Marco Colleoni (Italy)

- Giuseppe Curigliano (Italy)

- Suzette Delaloge (France)

- Carsten Denkert (Germany)

- Peter Dubsky (Switzerland)

- Bent Ejlertsen (Denmark)

- Eun Sook Lee (South Korea)
- Florian Fitzal (Austria)

- Prudence Francis (Australia)

- Viviana Galimberti (Italy)

- Heba Gamal (Egypt)

- Judy Garber (USA)

- Michael Gnant (Austria)

- William J. Gradishar (USA)

- Bahadir Gulluoglu (Turkey)

- Nadia Harbeck (Germany)

- Chiun-Sheng Huang (Taiwan)

- Jens Huober (Germany)

- Andre llbawi (WHO Cancer Control Program)

- Steven Johnston (UK)

- Sibylle Loibl (Germany)

- Monica Morrow (USA)

- Ann H. Patridge (USA)

- Martine Piccart (Belgium)

- Philip Poortmans (Belgium)

- Aleix Prat (Spain)

- Meredith Regan (USA)
- Isabella Rubio (Spain)

- Hope Rugo (USA)

- Emiel J.T. Rutgers (Netherlands)

- Felix Sedlmayer (Austria)

- Vladimir Semiglazov (Russia)

- Zhiming Shao (PR China)

- Tanja Spanic (Europa Donna)

- Petra Tesarova (Czech Republic)

- Beat Thürlimann (Switzerland)

- Sergei Tjulandin (Russia)

- Masakazu Toi (Japan)

- Maureen Trudeau (Canada)

- Nicholas Turner (UK)

- Inez Vaz Luis (France)

- Giuseppe Viale (Italy)

- Toru Watanabe (Japan)

- Walter Weber (Switzerland)

- Eric P. Winer (USA)

- Binghe Xu (PR China)

- Jiang Zefei (PR China) genes, such as ATM, BARD1, BRIP1, CDH1, CHEK2, NBN, PALB2, PTEN, STK11, RAD51C/RAD51D and TP53.

The German experts agree [1]. The AGO Mamma differentiates between mutations with high and moderate risk of disease. The clinical benefit of genetic testing is highest for the high-risk $B R C A 1 / 2$ genes (LoE $1 \mathrm{bA} \mathrm{AGO++),} \mathrm{as} \mathrm{it} \mathrm{results} \mathrm{in} \mathrm{effective} \mathrm{preven-}$ tive measures. The AGO has upgraded PALB2 mutations in terms of cumulative risk of disease (LoE 1bA AGO++). However, the efficacy of preventive measures is less well established for PALB2 mutations, which is why the recommendation regarding the clinical benefit of PALB2 mutation analysis was rated less strong (LoE 3aB $A G O+$ ). The moderately penetrant genes mentioned (ATM, BARD1, CHEK2, RAD51C, RAD51D) are among the core genes of common panels and will therefore usually be analysed as well. Clinical consequences should preferably be studied in the context of prospective trials or clinical registries (LoE 3aB AGO+/-) [1].

\section{Prophylactic mastectomy in confirmed PALB2 mutation?}

Half of the SG-BCC panellists consider prophylactic mastectomy to be justified in PALB2 mutation. From the German perspective, the high risk of the disease justifies prophylactic mastectomy, even though there is currently no data regarding the impact on survival. Due to the limited data available, competent counselling in a specialised centre should be pursued.

\section{Testing for adjuvant olaparib?}

PARP (poly ADP-ribose polymerase) inhibition is an effective treatment option in metastatic breast cancer with germline BRCA1/2 mutation (gBRCA1/2). In early breast cancer, the OlympiA trial [3] (NCT02032823) with the PARP inhibitor olaparib has reached its primary endpoint according to a press release. The scientific data will be presented at the 2021 annual meeting of the American Society of Clinical Oncology (ASCO). The SG-BCC panellists (56\%) voted in favour of future genetic testing of all patients eligible for adjuvant treatment with olaparib. The German experts agree in principle with the majority vote.

\section{Procedure in confirmed disease-causing mutation}

Drawing on fictitious cases with different clinical scenarios, the SG-BCC panellists discussed how to counsel patients at genetically increased risk of breast cancer.

- If a disease-causing mutation in BRCA1, BRCA2 or PALB2 - high penetrance (odds ratio $[O R]>3$ ) were detected, almost $85 \%$ of SG-BCC panellists would advise a 40 -year old woman to undergo risk-reducing bilateral mastectomy. For 60-year old women, barely half the SG-BCC panellists (46\%) voted in favour of this measure.

The German experts recommend that the decision-making process include a thorough counselling. Risk-reducing mastectomy is an effective procedure. Regular radiological monitoring, including MRI, may be an option. In some older women, prophylactic tamoxifen may also be an option [1]. 
- If a disease-causing mutation of intermediate penetrance (OR 2-3) was detected in the genes BARD1, CHEK2, CDH1, TP53, two-thirds of the SG-BCC panellists would recommend intensified screening, including MRI, for the 40-year-old woman, compared with only $42 \%$ of the SG-BCC panellists for the 60 year-old woman. A good third (35\%) consider routine monitoring to be adequate in older women.

- If disease-causing mutations were detected in the low-risk genes ATM, BRIP1, NF1, RAD51C, RAD51D, FRANCC, STK11 (low penetrance; OR 1-2), $50 \%$ of the SG-BCC panellists voted for intensified screening, including MRI, for the 40-year-old woman, while $40 \%$ considered routine monitoring to be adequate. In the 60-year-old woman, the SG-BCC panellists voted by majority (62\%) for routine breast screening. Only $30 \%$ recommended intensified monitoring, including MRI.

The German experts agree with the SG-BCC majority votes on the genes with medium or low penetrance. The lower the penetrance and the higher the age of the patient, the less aggressive the screening should be.

\section{Pathology}

\section{Relevance of the proliferation index (Ki-67)}

For years, the significance and validity of Ki-67 testing for treatment decisions in early oestrogen receptor-positive (ER+) and HER2-negative (HER2-) breast cancer have been discussed. Recently, an international working group [4] recommended Ki-67 testing, stating that patients with early ER+/HER2- breast cancer (T1-2 N0-1) and Ki-67 $\leq 5 \%$ do not need adjuvant chemotherapy, whereas Ki-67 $\geq 30 \%$ would warrant chemotherapy. Almost two thirds (62\%) of SG-BCC panellists agreed with this statement.

The German experts basically agree with the SG-BCC vote, but points out that the question involves extreme "cut-off" values with high "inter-observer" concordance [4,5-7].

For patients with ER+/PR+/HER2- breast cancer without lymph node involvement (N0), according to the SG-BCC vote (42\%), a Ki67 level of $30 \%$ and above indicates a high risk and thus the need for chemotherapy. The German experts do not agree with this majority vote, but agrees with those panellists (36\%) who state that no definitive Ki-67 cut-off in this situation exists (N0) indicating chemotherapy per se. For intermediate Ki-67 levels between 10 and $25 \%$, it is also necessary from the German point of view to include other criteria for risk assessment.

\section{Ki-67 measurement before and during neoadjuvant endocrine therapy}

Almost two thirds of the SG-BCC panellists (61\%) and the German expert group agree that $\mathrm{Ki}-67$ testing should be performed in routine clinical practice for all patients with early ER+/HER2- breast cancer. Likewise, two thirds (68\%) of SG-BCC panellists recommend Ki-67 testing during or after neoadjuvant endocrine therapy (NET) to assess treatment response. They also voted by a majority (70\%) that the prognosis of patients with ER+/HER2- ductal breast cancer can be assessed by the change in Ki-67 levels after a 2-week course of endocrine therapy (NET). The German experts agree that a 2-4 week NET to assess endocrine sensitivity, as used
- Table 2 In early ER+/PR+/HER2- breast cancer (T 1-3 cm), the multigene signature is recommended by the SG-BCC panel in selected cases. Consent of the German experts.

\begin{tabular}{|c|c|c|c|}
\hline \multirow[t]{2}{*}{ Patient profile } & \multicolumn{3}{|c|}{ Gene expression analysis } \\
\hline & Never & $\begin{array}{l}\text { In selected } \\
\text { patients }\end{array}$ & Routine \\
\hline \multicolumn{4}{|l|}{ Gender } \\
\hline - Male & & $x(56 \%)^{*}$ & \\
\hline - Female & & $x(72 \%)$ & \\
\hline \multicolumn{4}{|l|}{ Menopause status } \\
\hline - Premenopausal & & $x(67 \%)$ & \\
\hline - Postmenopausal & & $x(64 \%)$ & \\
\hline \multicolumn{4}{|l|}{ Nodal status } \\
\hline - Negative (N0) & & $x(63 \%)$ & \\
\hline $\begin{array}{l}\text { - 1-3 involved lymph } \\
\text { nodes }\end{array}$ & & $x(83 \%)$ & \\
\hline $\begin{array}{l}\text { - } \geq 4 \text { involved lymph } \\
\text { nodes }\end{array}$ & $x(79 \%)^{*}$ & & \\
\hline \multicolumn{4}{|l|}{ Tumour grading } \\
\hline - G1 & & $x(60 \%)$ & \\
\hline - G2 & & $x(72 \%)$ & \\
\hline . G3 & & $x(61 \%)$ & \\
\hline
\end{tabular}

for example in the German ADAPT trial [8] and in the POETIC trial [9], is an appropriate measure [1].

\section{Focus on multigene signatures}

In certain circumstances, multigene signatures can support the treatment decision for/against chemotherapy in early ER+/HER2breast cancer. Based on various clinical scenarios, the SG-BCC panellists voted on when multigene signatures would be helpful. The starting point in each case is a patient with early ER+/HER2breast cancer (tumour size $1-3 \mathrm{~cm}$ ) eligible for chemotherapy. The clinical situation of this patient varied in terms of gender (male/female), menopausal status and age (pre/postmenopausal), axillary lymph node (LN) involvement (pN0, 1-3 LN, $\geq 4 \mathrm{LN}$ ), and tumour grading (G1, G2, G3).

The majority of SG-BCC panellists recommended gene expression analysis in selected patients ( $\triangleright$ Table 2 ). A majority of the panellists $(79 \%)$ rejected multigene signature in patients with ER+/HER2- primary breast cancer $(1-3 \mathrm{~cm})$ with four or more affected lymph nodes.

The German experts basically agree and emphasise that because of the available prospective data gene expression analyses are only indicated in patients with a maximum of three affected lymph nodes. Moreover, gene expression analyses should only be performed if the decision for/against chemotherapy cannot be based on the usual clinical and pathological factors. The voting of the SG-BCC panellists are in line with the AGO recommendations [1]. In addition, the German experts point out the poor data in men, which is why the argument for them can only be made by 
analogy. In the context of the SG-BCC recommendation, it should be noted that in Germany the multigene testing recommended by the AGO is reimbursed for routine care in N0 patients, but in patients with LN involvement only under special contract arrangements (e.g., outpatient specialist management) [1].

\section{Early TNBC: no PD1/PD-L1 testing}

The SG-BCC panellists and the German experts agree that neither PD1/PD-L1 testing (majority vote: 93\%) nor tumour-infiltrating lymphocytes (TILs; majority vote: $61 \%$ ) are routinely indicated in patients with early triple-negative breast cancer (TNBC: ER-/PR -/HER2-) undergoing systemic treatment [1].

\section{Neoadjuvant systemic therapy}

\section{General considerations}

The concept of neoadjuvant therapy is recognised and favoured in Germany as a standard approach in early breast cancer as soon as adjuvant chemotherapy is indicated under the same treatment regimen [1]. In contrast, the SG-BCC panellists (60\%) do not favour the concept of neoadjuvant therapy (60\%). Pathological complete response ( $p C R$ ) following neoadjuvant systemic therapy (NAST) may be used as a surrogate endpoint for drug approval in early breast cancer. With a clear majority (83\%), the SG-BCC panellists put this approach into perspective: Achieving PCR is promising but inadequate when defining standard treatment. This can only be defined based on survival data. The German experts agree.

\section{NACT or NET?}

The SG-BCC panellists voted almost unanimously (98.21\%) that in postmenopausal patients with ER+/HER2- breast cancer and low risk, based on clinical pathological criteria or gene expression analysis, for whom neoadjuvant treatment is planned, endocrine therapy should be preferred over chemotherapy. The German experts agree that chemotherapy is not indicated here. In Germany, neoadjuvant endocrine therapy (NET) is not standard [1].

In early ER+/HER2- breast cancer, 74\% of SG-BCC panellists supported gene expression analysis of core biopsies to decide whether to administer NET or neoadjuvant chemotherapy (NACT). From the German perspective, the question - NET or NACT - is not significant clinically, as NET is not a standard regimen in Germany. Multigene signature is only reasonable in those patients where the indication for chemotherapy is questionable.

\section{Neoadjuvant systemic therapy in HER2 + breast cancer}

With a clear majority (85\%), the SG-BCC panellists saw no need for anthracyclines in addition to neoadjuvant taxane/anti-HER2based regimens in stage II breast cancer patients without LN involvement ( $\mathrm{cN} 0)$. In contrast, in $\mathrm{LN}$ involvement $(\mathrm{c} / \mathrm{pN}+)$, 54\% of SG-BCC panellists advocated neoadjuvant anthracycline/taxanebased chemotherapy plus anti-HER2 therapy.

In the modified case of a stage II/III cN0 patient with neoadjuvant taxane/trastuzumab therapy, $35 \%$ of the panellists voted to administer an anthracycline plus pertuzumab in addition to the neoadjuvant taxane/trastuzumab regimen. Just under 30\% (27\%) chose pertuzumab/platinum and $24 \%$ opted to add pertuzumab only.

The AGO Mamma [1] rates anthracycline-free and anthracycline-containing standard regimens in combination with trastuzumab plus pertuzumab as equally effective. However, various longterm sequelae have been described, and this needs to be discussed with the patient. The German experts therefore agree with the SG-BCC vote that $\mathrm{CNO}$ patients can receive a standard anthracycline-free regimen. This is also true for patients with $L N$ involvement regardless of the stage (for example: six cycles of TCbH [docetaxel, carboplatin, trastuzumab] or six cycles of TCbHP [TCbH + pertuzumab]). Some German experts, however, prefer treatment with anthracyclines in cases of higher risk, e.g., with lymph node involvement.

\section{Neoadjuvant therapy in TNBC}

In early TNBC, carboplatin is an effective treatment option alongside anthracyclines and taxanes. About $60 \%$ of SG-BCC panellists rejected neoadjuvant carboplatin in addition to anthracycline/cyclophosphamide/taxane-based treatment. The German experts see a difference to the German recommendations. According to the AGO Mamma [1], neoadjuvant platinum-containing chemotherapy can be used in early TNBC depending on patient risk profile and possible side effects (LoE 1aA AGO+). In Germany, carboplatin is usually combined with a taxane.

\section{No checkpoint inhibition in early TNBC}

The German experts agree with the SG-BCC panellists (90\%) that at present checkpoint inhibitors should not be given in early TNBC outside of clinical trials.1] Trial participation (GeparDouze, Alexandra, Neo Mono) is recommended [10-12].

\section{Local Treatment Following NAST}

\section{Residual axillary tumour}

The SG-BCC panellists (73\%) and the German experts agreed that axillary lymph node dissection (ALND) is indicated if a macrometastasis (> $2 \mathrm{~mm}$ ) was confirmed in the sentinel LN (SLN) or target LN (= biopsied and labelled LN; TLN) following NAST.

The majority of SG-BCC panellists see no indication for ALND if following NAST micrometastases ( $\leq 2 \mathrm{~mm}$ ) (majority vote: $60 \%$ ) and isolated tumour cells (ypNO[i+]) are detected only in the SLN (majority vote: $89 \%$ ). $72 \%$ of SG-BCC panellists recommend ALND if $1 / 3$ of the $S L N$ is affected. The German experts agree in principle, but refers to the differentiated recommendations of the AGO Mamma [1] ( $\triangleright$ Fig. 1). If a SLN or the TLN is "positive" following NAST, ALND is indicated irrespective of the number of LN examined and the size of the detected metastasis. There is agreement that axillary dissection is not justified when only isolated tumour cells are detected (ypNO[i+]).

The German experts criticise that the issues voted on do not contain any information on the axillary status before NAST. They add that ALND, just like SLND or TAD ("targeted axillary dissection”, i.e., TLN plus SLN excision [SLNE]), also serve diagnostic objectives. At present, however, there is no evidence for superiority of a regional therapy option (ALND vs. radiotherapy) in patients 


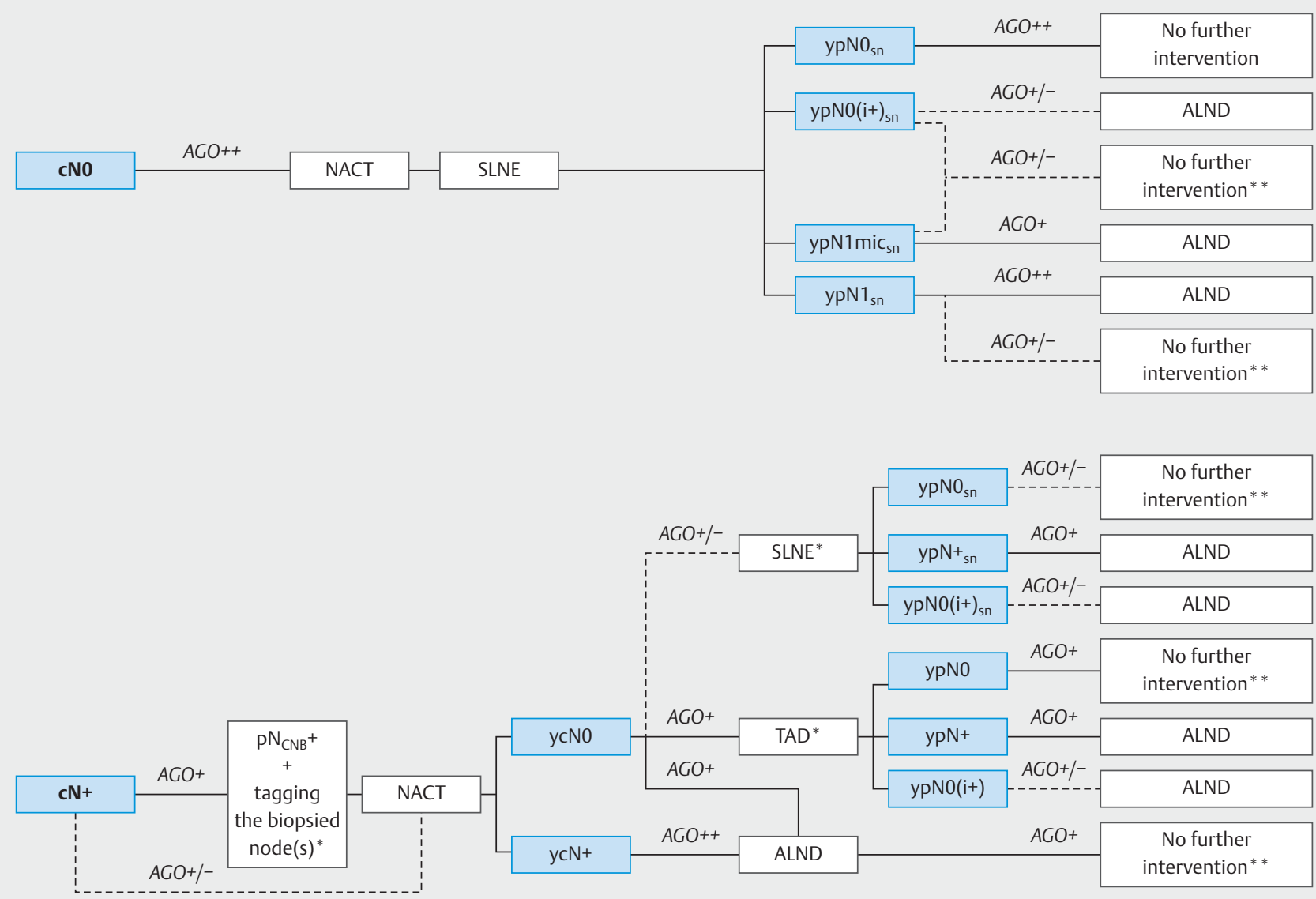

ALND: axillary lymph node dissection, CNB: core needle biopsy, NACT: neoadjuvant chemotherapy, SN: sentinel node, SLNE: sentinel lymph node excision, TAD: targeted axillary dissection (SLNE + TLNE), TLNE: targeted lymph node excision

${ }^{*}$ Participation in AXSANA trial recommended, ${ }^{* *}$ For radiotherapy procedure, see recommendations for radiotherapy

- Fig. 1 Recommendations of the AGO Mamma on the surgical approach in the axilla following neoadjuvant chemotherapy, from: [1]. Source: Courtesy of AGO Mamma.

with ycN0 or ypN1 status. Basically, according to the German experts, the evidence is limited and partly based on empirical data. The AXSANA/EUBREAST 3 trial [13] undertaken by the AGO Breast study group and the AWOgyn (Arbeitsgemeinschaft für ästhetische, plastische und wiederherstellende Verfahren in der Gynäkologie e.V.) will close these gaps in knowledge.

\section{Is it possible to omit ALND?}

There was no consensus SG-BCC vote on the question of whether ALND can be avoided in patients with positive nodal status (cN1) before treatment and marked, histologically positive TLN who convert to ycNO status and whose lymphatic drainage areas (LDA) must be irradiated. $41 \%$ of SG-BCC panellists agreed to omit ALND when $3 / 3$ SLNs were tumour-free and $37 \%$ of panellists agreed when $1 / 1$ SLN was tumour-free.

From the German perspective, the question cannot be answered because it is unclear whether the TLN had been removed.
The evidence on this is sparse. Although the German experts emphasise that the AGO recommends both: TAD and ALND in the situation of ycN0. If the TLN corresponds to the SLN, a negative SLN suffices $[14,15]$. More than $80 \%$ of SG-BCC panellists $(82 \%)$ recommend ALND when a patient with cN1 status (biopsy confirmed) has not responded or has only responded marginally to NAST (ypN1). For this situation (ypN+), the AGO Mamma recommends ALND ( $\triangleright$ Fig. 1) [1].

\section{Patient with unsuspicious nodes at presentation (cNO) with positive SLN following NAST}

The SG-BCC panellists voted on whether radiotherapy of the axilla can replace ALND in selected patients with initial cN0 but positive SLN (ypN1).

- The majority of SG-BCC panellists (62\%) recommend ANLD instead of radiotherapy in patients with $2 / 3$ "positive" SLN, if there was at least one macrometastasis (> $2 \mathrm{~mm}$ ). 


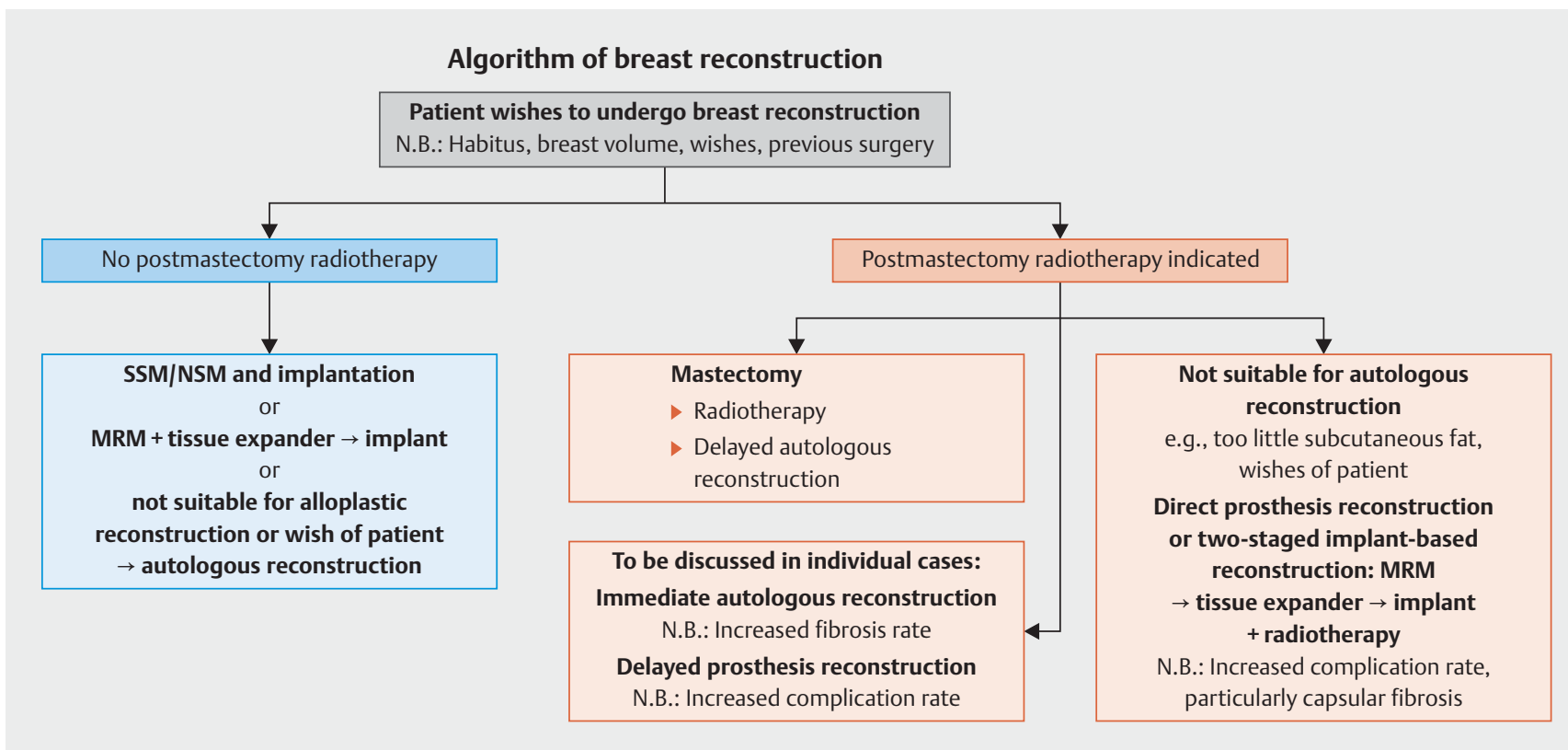

- Fig. 2 AGO Mamma algorithm for breast reconstruction following mastectomy, from: [1]. Source: Courtesy of AGO Mamma.

- If only $1 / 3$ SLN demonstrates macrometastasis (> $2 \mathrm{~mm}$ ), $48 \%$ of SG-BCC panellists voted for axillary radiotherapy; $52 \%$ voted for ALND.

- When ypN1mic $(>0.2-2 \mathrm{~mm})$ or ypNo(i+) $(\leq 0.2 \mathrm{~mm})$ was detected in $1 / 3 \mathrm{SLN}$, the majority of SG-BCC panellists ( $72 \%$ and $88 \%$, respectively) favoured axillary radiotherapy over ALND.

The more residual tumour following NAST, the more SG-BCC panellists felt that level $\mathrm{I} / \mathrm{II}$ radiotherapy alone did not have an adequate therapeutic effect, and therefore ALND should be performed. The German experts agree in principle, but refer to the markedly more differentiated AGO recommendations ( $\mathbf{F i g . 1 )}$ [1]. Due to the limited evidence, the AGO Mamma still recommends ALND. There is an increased risk of additional lymph node involvement following NAST and that - unlike in primary surgery $[16,17]$ - these are treatment-resistant cells with questionable response to radiotherapy. Due to the poor data in general, new recommendations must be postponed until the ongoing trials [13, 16,18 ] have been completed. Overall, the SG-BCC questions on LN staging following NAST do not reflect the complexity of the situation.

\section{Axillary intervention regardless of subtype}

Neither the tumour subtype nor the respective available postneoadjuvant treatment options in patients with positive LN following NAST affect the need for ALND. The majority of SG-BCC panellists rejected omitting ALND in favour of SLNE plus radiotherapy. The German experts agree, as there is no data that the decision for ALND following NAST depends on morphology or intrinsic subtype.

\section{TAD following NAST?}

The surgical approach in the axilla following NAST does not depend on tumour biology. The German experts agree with the respective majority vote of the SG-BCC panellists on the TAD indication - assuming optimised and standardised technique [13].

- TAD is an adequate alternative to standard ALND (60\%).

- TAD is an option in c/pN1 patients with conversion to ycN0 (90\%).

- TAD is an option regardless of breast cancer subtype (85\%).

Beside that "no surgery" is not an option in cases of presumed pathological complete remission, no new statement on breast surgery following NAST was presented at the SG-BCC this year. From the German perspective, however, it should be noted that surgery in the new, shrunken tumour volume remains standard according to the AGO Mamma, thus facilitating a very high breast conserving surgery rate [1].

\section{Surgery, Radiotherapy and Breast Reconstruction}

\section{Management following mastectomy}

On the question of how and when patients should undergo postmastectomy radiotherapy (PMRT), 32\% of SG-BCC panellists voted for an expander during PMRT (before planned reconstruction), while $20 \%$ would irradiate first and reconstruct later. The remainder favoured immediate reconstruction with autologous tissue (25\%) or an implant (single- or two-stage procedure; $23 \%$ ).

From the German perspective, all other options are also possible and should be discussed by the tumour board and with the pa- 
tient $(\mathrm{AGO}++)$ [1]. Delayed autologous reconstruction is preferred, with temporary expander or implant if needed. > Fig. 2 illustrates the breast reconstruction algorithm recommended by the AGO Mamma [1]. The decision for breast reconstruction, especially delayed reconstruction, must be discussed with each patient individually. The German experts note an increased risk of complications (e.g., risk of capsular fibrosis) if radiotherapy is performed after implant reconstruction.

\section{Hypofractionated radiotherapy following reconstruction?}

If a patient with immediate breast reconstruction requires PMRT, the SG-BCC panellists (64\%) without restrictions consider moderate hypofractionated radiotherapy a suitable option. The German experts note the clearly limited data available. The AGO Mamma has not commented on hypofractionated PMRT [1].

\section{Radiotherapy Following Breast-Conserving Surgery}

\section{Hypofractionated radiotherapy as standard?}

In stage I/II ER+/HER2- breast cancer following breast-conserving surgery (BCS) with negative resection margins, the majority of SG-BCC panellists (72\%) voted for moderately hypofractionated whole-breast radiotherapy (WBRT; 15-16 fractions) as the preferred fractionation regimen, regardless of patient age. This is consistent with the $\mathrm{S} 3$ guideline and the recommendation of the AGO Mamma [1,2]. 9\% of the SG-BCC panellists favoured ultrashort course WBRT, in line with the FAST and FAST-Forward trials $[19,20]$. The AGO Mamma defines ultra-short course WBRT as an alternative in selected cases ( $\mathrm{LoE} 1 \mathrm{bB} \mathrm{AGO}+/-$ ) when standard hypofractionated WBRT is not an option [1]. This is supported by a statement of the German Society of Radiation Oncology (DEGRO) [21]. Ultra-short course radiotherapy may become the treatment of the future.

\section{Focus on partial breast irradiation}

For partial breast irradiation (PBI) in stage I/II ER+/HER2- breast cancer without LN involvement, the majority of SG-BCC panellists saw no PBI indication in patients with lobular breast cancer (80\%), or lymphovascular invasion (87\%), or germline mutation (85\%), and/or in patients $<40$ years (92\%). The German experts agree in principle, but notes the limited data available, especially on germline mutations, which is why there is no guideline recommendation $[1,22]$. However, the increased risk of ipsilateral secondary cancer due to genetic predisposition and the protective effect of WBRT support the recommendation [23].

\section{Importance of multigene signatures in radiotherapy}

According to the SG-BCC majority vote, commercially available multigene signatures do not provide a basis for deciding whether regional nodal irradiation (RNI: 92\%) or chest wall irradiation $(89 \%)$ is indicated. This also applies to the decision to forego radiotherapy in invasive breast cancer following breast-conserving surgery (84\%). The German experts agree in each case. The AGO
Mamma [1] and DEGRO [24] advise against the use of multigene signatures in these situations.

\section{Post-neoadjuvant Systemic Therapy}

\section{General consideration}

The prognostic significance of pathological complete response (pCR: ypT0/is pN0) following neoadjuvant chemotherapy (NACT) is undisputed. The question of whether the tumour stage at initial diagnosis or the intrinsic tumour subtype also affect the future outcome of a patient with PCR was supported by two-thirds of the SG-BCC panellists (65\%). The German experts agree and add that the prognosis following NACT can be estimated with different models [25-27].

\section{Post-neoadjuvant therapy in HER2 positive breast cancer}

For patients with HER2+ breast cancer and clinically suspect lymph nodes at initial diagnosis ( $\mathrm{cN}+$ ) who achieve $\mathrm{PCR}$ with neoadjuvant chemotherapy plus trastuzumab and pertuzumab, the SG-BCC panellists (56\%) recommend continuing post-neoadjuvant treatment with trastuzumab and pertuzumab. If the patient was CN0 at initial diagnosis, the SG-BCC panellists (70\%) recommend that additional administration of pertuzumab is not needed in the post-neoadjuvant setting. The German experts agree with both majority votes. The AGO Mamma recommends post-neoadjuvant trastuzumab ( $\mathrm{LoE} 2 \mathrm{aC} \mathrm{AGO}++$ ) in low risk of recurrence patients and additional pertuzumab in high risk patients (cN+: $\mathrm{LoE}$ 2bC $\left.\mathrm{AGO}^{+}\right)[1]$.

If a patient does not achieve pCR after standard NAST, further post-neoadjuvant treatment with trastuzumab emtansine (T-DM1) is the therapy of choice for almost all SG-BCC panellists (89\%). This corresponds to the AGO recommendation (LoE $1 \mathrm{bB}$ $\mathrm{AGO}+$ ). According to the SG-BCC majority vote (77\%) and the German experts, post-neoadjuvant T-DM1 is also indicated in small tumour residuals $(<5 \mathrm{~mm})$ [28].

\section{Post-neoadjuvant therapy in TNBC}

In patients with early TNBC and pCR following NACT plus immunotherapy, the SG-BCC panellists (85\%) see no indication for post-neoadjuvant checkpoint inhibitors. The German experts agree. No survival data from clinical trials are available yet.

If patients who do not achieve PCR, continued treatment with capecitabine is a post-neoadjuvant option. The German experts agree with the majority vote of the SG-BCC panellists (88\%). The AGO-Mamma recommends up to eight cycles of capecitabine (LoE 1bB AGO+). Trial enrollment is recommended (LoE 5D AGO +) [1], for example, in the SASCIA trial [29].

\section{Post-neoadjuvant therapy in ER+/HER2- breast cancer}

The SG-BCC panellists stated unanimous (100\%) that patients with early, hormone-sensitive (ER+/HER2-) breast cancer who have not achieved $\mathrm{PCR}$ in the breast but a good response in the axilla (pN0) after NET should receive post-neoadjuvant chemotherapy. The German experts agree with the statement, with the comment that conventional (4-6 months) NET is rarely used in 


\begin{tabular}{|c|c|c|c|}
\hline \multirow{2}{*}{$\begin{array}{l}\text { Radiotherapy (RT) after breast conserving surgery (invasive cancer) - } \\
\text { boost irradiation }\end{array}$} & \multicolumn{2}{|c|}{ Oxford } & \multirow[b]{2}{*}{ AGO } \\
\hline & LoE & GR & \\
\hline \multicolumn{4}{|l|}{ Boost-RT (improves local control, no survival benefit) } \\
\hline Premenopausal & $1 \mathrm{~b}$ & B & ++ \\
\hline $\begin{array}{l}\text { Postmenopausal, if >T1* }, \mathrm{G} 3, \mathrm{HER} 2 \text {-positive, triple-negative, EIC } \\
\text { (at least } 1 \text { factor) }\end{array}$ & $2 b$ & B & + \\
\hline \multicolumn{4}{|l|}{ Techniques } \\
\hline Percutaneous (photons, electrons) as sequential boost & $1 \mathrm{a}$ & A & ++ \\
\hline Multicatheter brachytherapy boost & $1 \mathrm{a}$ & A & ++ \\
\hline $\begin{array}{l}\text { Percutaneous boost as simultaneous integrated boost } \\
\text { (with conventionally fractionated whole-breast irradiation) }\end{array}$ & $1 b$ & B & + \\
\hline $\begin{array}{l}\text { Percutaneous boost as simultaneous integrated boost } \\
\text { (with hypofractionated whole-breast irradiation) }\end{array}$ & $2 b$ & B & $+1-$ \\
\hline Intraoperative boost irradiation (followed by whole-breast irradiation) & $2 b$ & B & + \\
\hline
\end{tabular}

- Fig. 3 Recommendations of the AGO Mamma on "boost" irradiation following BCS in invasive breast cancer, from: [1]. Source: Courtesy of AGO Mamma.

Germany and is primarily reserved for older patients or those with significant comorbidities.

\section{No pCR following NET}

Further SG-BCC questions on post-neoadjuvant chemotherapy in patients (ER+/HER2-) without pCR following NET rarely arise in Germany, as NET is not a standard treatment regimen in Germany. Moreover, the probability of pCR following NET is very low (5\%).

\section{Ductal Carcinoma in Situ (DCIS)}

\section{Postoperative radiotherapy in ER+ DCIS?}

According to the SG-BCC panellists (majority vote: $58 \%$ ), omitting postoperative radiotherapy following BCS of ER+ DCIS with adequate resection margin is justified in all patients over 70 years of age, and in principle (no age limit in the question) in patients at low biological ("luminal-like”) or genomic (multigene testing) risk (70\%) as well as in low grade tumours (G1, 74\%). Two-thirds of the SG-BCC panellists $(67 \%)$ recommend omitting postoperative radiotherapy only in older patients (> 70 years) with at least one of the low-risk factors noted above. A simple majority (53\%), on the other hand, saw an indication for postoperative radiotherapy in unifocal DCIS $(\leq 2 \mathrm{~cm})$ without necrosis.

The German experts point out that postoperative radiotherapy following BCS of ER+ DCIS with adequate free resection margin reduces the recurrence rate in the affected breast, but has no effect on overall survival [30]. This should be discussed with each patient individually. In the view of the Germans group, the voting results reflect that the overall risk profile should be taken into account when deciding for or against radiotherapy following BCS in DCIS.

\section{Endocrine therapy following BCS and R0 situation}

Various options are available if a patient with ER+ DCIS and postoperative radiotherapy also desires endocrine therapy as recurrence prophylaxis [1]. The vast majority of the panel favoured endocrine therapy (83\%) with standard-dose $(20 \mathrm{mg} /$ day) or lowerdose (5 mg/day) tamoxifen or an aromatase inhibitor (Al).

The AGO Mamma recommends endocrine therapy in addition to adjuvant radiotherapy as an option in individual cases $\left(\mathrm{AGO}+{ }^{-}\right)$. All the choices listed (tamoxifen $20 \mathrm{mg}$, tamoxifen $5 \mathrm{mg}, \mathrm{Al}$ [only in postmenopausal patients]) are an option [1]. The German experts emphasise that the indication for endocrine therapy depends on possible risk factors, potential side effects and the patient's wishes. The patient should be informed that endocrine therapy is not associated with an overall survival benefit, but may in particular reduce the risk of secondary cancer in the other breast (LoE 1a) [1].

\section{Radiotherapy}

\section{Importance of the boost}

The SG-BCC panel heterogeneously discussed the issue of routine boost irradiation following BCS and WBRT in patients with early invasive breast cancer. Nearly half (47\%) would boost the tumour bed only in younger patients ( $18 \%$ in $<40$ years, $29 \%$ in $<50$ years), $31 \%$ would base their indication on tumour biology alone (G3, extensive intraductal component, HER2-positive, TNBC), while $20 \%$ regard the boost as indicated in every patient. From the German perspective, the heterogeneous vote is also reflected in the recommendations of the AGO Mamma ( $\vee$ Fig. 3) [1]. The AGO Mamma clearly recommends the boost in premenopausal patients ( $\mathrm{LoE} 1 \mathrm{bB} \mathrm{AGO}++)$. In postmenopausal patients, boost is only indicated in patients at increased risk (LoE 2bB AGO+) [1]. 
Radiotherapy following NACT

\begin{tabular}{|c|c|c|c|c|c|c|c|}
\hline Pretreatment & Posttreatment & RT-BCS & PMRT & RT-RN* & LoE & GR & AGO \\
\hline Locally advanced & $\mathrm{pCR} /$ no $\mathrm{pCR}$ & Yes & Yes & Yes & 1a/1a/1a & $\mathrm{A} / \mathrm{A} / \mathrm{A}$ & $++|++|++$ \\
\hline $\mathrm{cT} 1 / 2 \mathrm{cN} 1+^{* *}$ & ypT1+ or ypN1+ (no pCR) & Yes & Yes & Yes & $1 a / 2 b / 2 b$ & $A / B / B$ & $++1+1+$ \\
\hline $\mathrm{cT} 1 / 2 \mathrm{cN} 1+^{* *}$ & ypT0/is ypN0 & Yes & \multicolumn{2}{|c|}{ Increased risk of relapse ${ }^{1}$} & $2 b / 2 b / 2 b$ & $\mathrm{~B} / \mathrm{B} / \mathrm{B}$ & $+1+1+$ \\
\hline cT1/2 cN0 (ultrasound mandatory) & ypT0/is ypNO & Yes & No & No & $2 b / 2 b / 2 b$ & $A / B / B$ & $+1-1-$ \\
\hline \multicolumn{8}{|l|}{ Local advanced: T3-4 or cN2-N3 } \\
\hline \multicolumn{8}{|c|}{$\begin{array}{l}{ }^{1} \text { Criteria for increased risk of relapse: } \\
\text { pN0 premenopausal high risk: central or medial tumor localization, and (G2-3 and ER/PR negative) } \\
\text { Pretreatment pN1a/cN+* high risk: central or medial tumor localization and (G2-3 or ER/PR negative) } \\
\text { or premenopausal, lateral tumor localization and (G2-3 or ER/PR negative) }\end{array}$} \\
\hline \multicolumn{8}{|c|}{$\begin{array}{l}\text { * Regarding coverage of axilla level I/II please also see slides "Additional RT of the axilla after primary surgery"/ } \\
\text { "Additional RT of the axilla after neoadjuvant therapy" }\end{array}$} \\
\hline ** confirmed by core biopsy & & & & & & & \\
\hline
\end{tabular}

- Fig. 4 Recommendations of the AGO Mamma on radiotherapy following neoadjuvant systemic chemotherapy, from: [1]. Source: Courtesy of AGO Mamma.

\section{Boost in DCIS?}

The SG-BCC panellists reject routine radiation boost both generally in DCIS (89\%) and also in low-risk DCIS patients (96\%). A slight majority $(55 \%)$ rejects routine radiation boost in DCIS patients $<50$ years of age. In contrast, two-thirds of SG-BCC panellists $(65 \%)$ recommend routine radiation boost in DCIS patients at increased risk, for example, due to necrosis, close resection margins $(<2 \mathrm{~mm})$, and large lesions.

The AGO Mamma recommends radiation boost in DCIS patients only in special cases at increased risk (LoE 1 bB AGO+/-). According to the AGO, this includes patients $<50$ years of age or those $\geq 50$ years if additional risk factors are present (e.g., symptoms, $\mathrm{G} 2 / 3$, central necrosis, close resection margins, multifocal tumour, etc.) [1]. The German experts point out that this is an individual decision that should be discussed in the multispecialty tumour board and with the patient. Data from a randomised trial [31] on this question was presented for the first time at the San Antonio Breast Cancer Symposium 2020 showing no difference between hypofractionated radiotherapy and conventional radiotherapy and a moderate advantage of adding the boost.

\section{Moderate hypofractionation in invasive cancer}

The SG-BCC panellists regard hypofractionation as adequate radiotherapy modality following mastectomy (90\%) and in regional nodal irradiation (RNI) (76\%). - The AGO Mamma has not issued a statement regarding fractionation in PMRT without RNI. For combined irradiation of the chest wall with RNI, the German experts recommend conventional fractionation due to the limited data available on hypofractionation.

The AGO Mamma regards hypofractionated RNI as an option in selected cases ( $\mathrm{LoE} 2 \mathrm{bB} A G O+/-$ ). The publication by Wang et al. [32] is based solely on patients with locally advanced breast cancer with a short follow-up period. In Germany, the standard is conventional fractionation over a period of five weeks ( $\mathrm{LoE} 1 \mathrm{aA} \mathrm{AGO}++$ ). Current ongoing trials will clarify this question [33-36].
The majority of SG-BCC panellists (59\%) voted in favour of hypofractionated radiotherapy as the standard option for the chest, thoracic wall and regional lymph nodes - only rare circumstances such as repeat (second) radiotherapy were excluded. Due to the limited data on hypofractionation, the AGO Mamma recommends conventional fractionated radiotherapy in this indication, but considers hypofractionated irradiation an option (LoE 2bB AGO+/-). Only $21 \%$ of SG-BCC panellists considered hypofractionated radiotherapy a standard option only following BCS, regardless of the patient's age. This vote corresponds to the recommendations of the AGO Mamma [1]. Regardless of this, it was pointed out at the SG-BCC that obstacles to the implementation of hypofractionation, for example, billing models based on the number of radiation fractions, should be reduced $[37,38]$.

\section{Regional node irradiation (RNI) following NAST}

Another focus of the SG-BCC vote was the importance of RNI following NAST in TNBC or HER2+ breast cancer stage II and above. In clinically unsuspicious LN before NACT (cN0), a clear majority of SG-BCC panellists saw no indication for RNI in PCR of the primary tumour (TNBC: 86\%; HER2+: 90\%). In contrast, in patients with stage I/III, pCR and clinically suspect LN (cN1) prior to NACT, $70 \%$ (TNBC) and 65\% (HER2+) routinely recommended RNI despite pCR. Only 26\% (TNBC) and 30\% (HER2+) restricted this to stage III patients.

With reference to the recommendation of the AGO Mamma, the German expert group emphasises that the indication for RNI should be risk-adapted ( $\triangleright$ Fig.4) [1]. They therefore fully agree with the SG-BCC vote in the CNO patient. In contrast, RNI is indicated in cN1 patients with initial stage III despite PCR. In stage II, the indication for RNI should be discussed with the patient depending on other risk factors. 


\section{Elderly patients with life expectancy $>10$ years}

According to the AGO Mamma, adjuvant radiotherapy can be omitted in patients with small invasive ER+/HER2- breast cancer (pT1pN0) who have undergone breast-conserving surgery and have a life expectancy of less than 10 years, after individual consultation and accepting an increased risk of intramammary recurrence. This requires that the cancer has been resected completely (RO) and that the patient receives adjuvant endocrine therapy $(\mathrm{LoE} 1 \mathrm{aB} \mathrm{AGO}+)[1]$.

The SG-BCC also discussed the question of adjuvant radiotherapy in older patients (> 70 years) with breast-conserving surgery in ER+/HER2- breast cancer and a life expectancy of more than 10 years. The majority of SG-BCC panellists did not recommend this option in general (90\%). The SG-BCC panellists (88\%) consider to omit additional radiotherapy, especially in patients with small ER+/HER2 - breast cancers $(<2.5 \mathrm{~cm})$ and low clinical or genomic risk. According to the SG-BCC majority vote, radiotherapy should not be omitted in larger tumours $(>2.5 \mathrm{~cm} / \mathrm{N0}$; majority vote: $80 \%)$, in the case of a positive SLN (90\%) and in the case of unfavourable clinical/biological factors or high genomic risk (92\%).

From the German perspective, the decision for or against adjuvant radiotherapy in older women with low-risk breast cancer requires an individual risk-benefit analysis. Trial data are so far only available with a follow-up period of up to about ten years [39]. Important options in older patients, which may affect the risk-benefit analysis in the future, are partial-breast radiotherapy and, potentially ultra-hypofractionated irradiation [1].

\section{Intraoperative radiotherapy}

Overall, $61 \%$ of SG-BCC panellists agreed that there are patients eligible for intraoperative radiotherapy (IORT) as sole modality. The German experts agree. The AGO Mamma considers intraoperative radiation alone to be a therapeutic option in patients $>70$ years of age with a low risk of recurrence (LoE $1 \mathrm{bA} \mathrm{AGO+}$ ) and in specific cases in patients $>50$ years of age $($ LoE $1 \mathrm{bA} \mathrm{AGO+/-)}$ [1]. Due to the methodical limitations of the TARGIT-A trial $[40,41]$, no general recommendation favouring IORT as sole modality can be given [22].

\section{Adjuvant Systemic Therapy in ER+ Breast Cancer}

\section{General considerations}

Half of the SG-BCC panellists defined the threshold of ER-positive cells detected by immunohistochemistry at $\geq 1 \%$ and $\geq 10 \%$ respectively. From the German perspective, the cut-off $\geq 1 \%$ is adequate for adjuvant endocrine therapy, although knowing that 1$10 \%$ ER-positive cells are considered as "questionably endocrine sensitive". Adjuvant endocrine therapy may be offered to these patients (LoE 3bD AGO+). With ER-positive cells $>10 \%$, there is a clear indication for treatment (LoE 1A AGO++) [1].

Patients with "questionably endocrine sensitive" ER+/HER2breast cancer must be informed accordingly. The German experts add that nowadays more sensitive antibodies are used in the detection of ER-positive cells and that it can be assumed that pa- tients with ER-low expression ("low expressers”) may have more biologically aggressive "basal-like" breast cancer.

The German experts and the SG-BCC panellists agree that patients with luminal A- or luminal B-like breast cancer without lymph node involvement (pN0) may benefit from adjuvant endocrine therapy regardless of tumour size - even in the case of microinvasion in the sentinel node (SG majority vote: 59 and $58 \%$, respectively).

In case of ER+/HER2+ breast cancer (pN0), the majority of SG-BCC panellists (51\%) voted in favour of adjuvant anti-HER2based therapy if the tumour size was $5 \mathrm{~mm}$ or larger. A minority of $15 \%$ would administer adjuvant anti-HER2-based therapy regardless of tumour size. - The AGO Mamma recommends the use of trastuzumab ( $\mathrm{LoE} 2 \mathrm{bB} \mathrm{AGO}+$ ) in pN0 patients and a tumour size $>5 \mathrm{~mm}$ and for tumour sizes $>10 \mathrm{~mm}$ (LoE 1aA AGO++). In tumours $\leq 5 \mathrm{~mm}$ in diameter, adjuvant trastuzumab is possible on an individual risk-adapted decision (LoE 2bB AGO+/-) [1].

\section{Duration of endocrine therapy in the premenopausal patient}

If a premenopausal patient with ER+/HER2- breast cancer and high risk of recurrence received adjuvant tamoxifen plus OFS (ovarian function suppression) for five years, almost $90 \%$ of SG-BCC panellists recommend continuing endocrine therapy for another five years. A slight majority (45\%) favour monotherapy with tamoxifen, $41 \%$ want to switch to an Al (plus OFS, if the patient remained premenopausal). Only four percent would continue tamoxifen/OFS.

The German experts emphasize that there is no valid data for extended adjuvant endocrine therapy (EAT) after five years of tamoxifen/OFS. From the German perspective, continued treatment with tamoxifen is an option. The AGO Mamma recommends continued treatment with tamoxifen (years 6-10) (LoE 1aA AGO++) after initial five years of tamoxifen and regards continued treatment with tamoxifen alone as a "possible" option (LoE 5D AGO+) after initial five years of endocrine therapy plus OFS [1].

\section{Duration of endocrine therapy in lymph node involvement}

According to the recommendation of the AGO Mamma, the standard is adjuvant endocrine therapy for five years (AGO++) [1]. For patients with lymph node involvement at initial diagnosis, the majority of SG-BCC panellists vote for endocrine therapy beyond five years: $34 \%$ recommend $7-8$ years and $53 \%$ would treat for a total of ten years. The AGO Mamma recommends treatment duration beyond five years after individual risk-benefit analysis (AGO++). Duration and choice of treatment and, if necessary, the sequence (Al or tamoxifen) depend, among other things, on patient menopausal status, treatment tolerance, risk of recurrence, and the patient's wishes [1].

\section{Adjuvant therapy with CDK4/6 inhibitors?}

CDK4/6 inhibitors have not yet been approved in Germany for adjuvant therapy in ER+/HER2- breast cancer. The only positive trial data currently available - albeit with a still short follow-up period (<20 months) - is for adjuvant abemaciclib from the monarchE trial [42]. In the SG-BCC vote, $54 \%$ of panellists favour abemaciclib 
in addition to adjuvant endocrine therapy in patients with at least four involved lymph nodes. In patients with 1-3 involved LN and additional risk factors (e.g. G3 and/or T3 or high Ki-67), an equally narrow majority of SG-BCC panellists (54\%) reject adjuvant abemaciclib. About $60 \%$ of the SG-BCC panellists do not see additional Ki-67 testing (in addition to other prognostic markers) as an option to allow patients the adjuvant treatment with a CDK4/6 inhibitor.

The AGO Mamma considers adjuvant abemaciclib over two years in addition to standard endocrine therapy to be an option in patients with an increased risk of recurrence and if the inclusion criteria of the monarchE study are met (LoE $2 \mathrm{bC} \mathrm{AGO+/-)} \mathrm{[1].}$

\section{Use of gene expression signatures}

The majority of SG-BCC panellists (79\%) reject the indication for chemotherapy in postmenopausal patients if their genomic risk according to the clinical criteria of the MINDACT [43], TAILORx [44] or RxPonder [45] or similar trials is low and/or their recurrence score (RS) is $\leq 25$.

The German experts agree in principle. Gene expression analysis should only be used if the traditional clinical-pathological factors (tumour size, nodal involvement, grading, Ki-67, ER/PR as well as HER2) do not allow decision-making for or against chemotherapy followed by endocrine therapy (vs. endocrine therapy alone). If gene expression analysis is indicated, the recommendation resulting from the analysis should be followed. In addition, the German experts refer to the current data of the ADAPT trial [8] as well as to the ADAPTlate [46] and ADAPTcycle [47] trials on this issue, which are currently recruiting patients with intermediate and higher clinical risk.

\section{Focus on OFS}

Standard adjuvant endocrine therapy in premenopausal patients $(\mathrm{ER}+/ \mathrm{HER} 2-)$ is tamoxifen for 5 years (AGO $1 \mathrm{aA}++)$ if the risk of recurrence is low, plus OFS ( $\mathrm{LoE} 2 \mathrm{bC} \mathrm{AGO}++$ ) in higher risk of recurrence. Tamoxifen/OFS treatment should only be given as long as it is tolerated by the patient and she is clearly premenopausal. According to the AGO recommendation, tamoxifen/OFS or Al/OFS is an option following chemotherapy once ovarian function returns within 24 months [1].

The German experts do not agree with the majority vote of the SG-BCC panellists recommending in principle an OFS in premenopausal patients with clinical stage II (71\%). In patients $<40$ years of age, as many as $94 \%$ would expand treatment with OFS. From a German perspective, the issue is not differentiated enough. The indication for OFS is based on the risk of recurrence.

If a patient with stage II ER+/HER2 breast cancer is premenopausal after initial chemotherapy, the question of further endocrine therapy was adressed. $43 \%$ of SG-BCC panellists see the indication for OFS (plus tamoxifen) in all patients, while $52 \%$ rely on supplementary OFS only in "high risk" cases (age < 40 years, lymph node involvement [ $\mathrm{N}+]$, high Ki-67 and/or luminal B carcinoma, or intermediate or high risk according to gene expression analysis). In a separate vote, $94 \%$ of the SG-BCC panellists favour OFS in principle as part of endocrine therapy in patients with a risk of recurrence that justifies the indication for chemotherapy, as long as the patient remains premenopausal.
From the perspective of the German experts, the voting results reflect that, regardless of the stage, it must be differentiated between patients with low and high risk of recurrence and that an OFS is only indicated with increased risk [1]. The previous chemotherapy is a surrogate marker for high risk.

\section{Role of multigene signatures in endocrine therapy}

In premenopausal patients with ER+/HER2- breast cancer without lymph node involvement and with low/intermediate genomic risk, e.g. RS $16-25$, the majority of SG-BCC panellists (53\%) agree on OFS in addition to tamoxifen or an Al. Almost a quarter recommended only tamoxifen (22\%) or endocrine therapy plus chemotherapy (24\%).

The German experts cannot completely agree with the majority vote. According to the AGO Mamma [1], chemotherapy plus endocrine therapy can be useful in this group with individually increased risk of recurrence [44]. This must be discussed with the informed patient and decided individually.

In patients with 1-3 positive LN and low genomic risk (e.g., RS $\leq 25$ ), $30 \%$ of SG-BCC panellists recommend chemotherapy followed by oral endocrine therapy, while $17 \%$ refuse oral endocrine therapy plus OFS. About one quarter consider both treatment options to be adequate, and $26 \%$ would prefer chemotherapy or endocrine monotherapy.

With regard to the indication for OFS, the German experts add that this should be independent of the genomic risk. So far, there is no data clearly proving a correlation between OFS indication and multigene test result.

\section{Oestradiol level during OFS?}

For patients under OFS, 53\% of SG-BCC panellists recommend routine measurement of oestradiol levels, while $47 \%$ reject this step. From the German perspective, there is no reason for routine testing. This corresponds to the $50: 50$ vote of the panellists. Testing should be done after hysterectomy to determine menopausal status and may also be useful during endocrine therapy with an $\mathrm{Al}$ plus $\mathrm{GnRH}$ analogue to verify endocrine suppression.

\section{Chemotherapy effect in premenopausal patients}

Chemotherapy efficacy in premenopausal patients is based not only on the cytotoxic effect but also on the ovarian suppression induced by chemotherapy - especially in patients with favourable biological factors (positive ER/PR status, well-differentiated cancer, low Ki-67 score, low genomic risk). The extent of an endocrine effect of the chemotherapy itself is under discussion. The voting result of the SG-BCC panellists was quite heterogeneous. From the German perspective, it is impossible to differentiate between cytotoxic and chemotherapy-induced endocrine effects.

\section{Effective chemotherapy regimens}

In patients with stage I/II ER+/HER2 breast cancer without LN involvement and chemotherapy indication, the majority of SG-BCC panellists (34\%) recommend an anthracycline/cyclophosphamide/taxane-based regimen, plus $6 \%$ who chose a dose-dense anthracycline-containing regimen. An anthracycline-free regimen with either four (32\%) or six cycles (12\%) of taxane/cyclophosphamide (TC) was favoured by $44 \%$ of panellists. The broad vote is in 
line with the recommendations of the AGO Mamma [1]. The German experts refer to the standard treatments recommended by the AGO Mamma [1]. The chemotherapy regimen to be used must be decided individually with the patient taking into account potential adverse events. In patients with low volume LN involvement, anthracycline-free standard chemotherapy regimens are generally considered to be equivalent to anthracycline-containing standard regimens. Equal efficacy with the standard anthracycline-taxane sequences has been demonstrated for the TC regimen only if six cycles ( $6 \times$ docetaxel/cyclophosphamide) are administered.

\section{Focus on the postmenopausal patient}

Trials such as ADAPT [8], MINDACT [43], TAILORx [44] and RXPONDER [45] have studied the impact of endocrine therapy \pm chemotherapy in ER+/HER2- breast cancer. Based on the trial outcomes, the SG-BCC panellists see the indication for chemotherapy in addition to endocrine treatment (vs. endocrine treatment alone) in the majority of postmenopausal stage III patients, regardless of biomarkers (68\%) and in the case of large tumour volume, for example, N3 ( $\geq 10$ affected lymph nodes) or T3N2 (96\%). The German experts agree in each case here, since in the high-risk clinical situation there is the basic indication for chemotherapy.

When asked whether chemotherapy is indicated in the same patient - postmenopausal, stage III - with G1/2 cancer and lobular histology, 48\% SG-BCC panellists agree, while 52\% reject this recommendation. In case of low-risk G1 cancer with Ki- $67<10 \%$, $63 \%$ do not recommend chemotherapy. In terms of the RS, $61 \%$ of SG-BCC panellists reject chemotherapy for RS < 11, while $58 \%$ see an indication for chemotherapy for RS $<25$.

From the German perspective it is impossible to comment on the indication for chemotherapy due to the limited information in the question. This is also reflected in the ambivalent outcome of the voting. The German experts add that the decision on chemotherapy does not depend on histology (NST or lobular), but on the known clinical-pathological factors and, if needed, on gene expression analysis.

\section{Chemotherapy for high tumour burden}

If chemotherapy is indicated in ER+/HER2 negative breast cancer with locally advanced stage or with a high tumour burden, the German experts refer to the standard chemotherapies as recommended by the AGO Mamma [1].

\section{Adjuvant Systemic Therapy in Estrogen Receptor-negative (ER-) Breast Cancer}

\section{TNBC and ER-/HER2+ breast cancer}

In pNO patients with ER-negative (ER-) and HER2+ breast cancer and tumour size of 5-6 mm and larger, a majority of SG-BCC panellists (52\%) recommend adjuvant anti-HER2-based systemic therapy. Almost as many (46\%) would also start adjuvant antiHER2 therapy in smaller lesions (including $12 \%$ even in microinvasion). The German experts add that the prognostic data of this patient group reveals a significant risk of recurrence regardless of tu- mour size [48 - 50]. Recent retrospective data [51] suggests an effective effect of adjuvant anti-HER2 therapy even in very small HER2+ breast cancers (pT1a).

In case of TNBC without LN involvement (pN0), the majority of SG-BCC panellists (46\%) favour adjuvant systemic therapy if the tumour size is $5 \mathrm{~mm}$ or larger. The German expert group agrees with reference to the AGO Mamma [1].

\section{Adjuvant Systemic Therapy in HER2-positive Breast Cancer}

\section{Trastuzumab \pm Pertuzumab}

Patients with HER2+ breast cancer usually also require adjuvant anti-HER2 targeted treatment if chemotherapy is indicated. The AGO Mamma recommends trastuzumab-based adjuvant therapy in patients without $L N$ involvement and tumour size larger than 5 mm (6-10 mm: LoE 2bB AGO+; > 10 mm: LoE 1aA AGO++). This decision must be re-evaluated on a case-by-case basis in HER2+ breast cancer $\leq 5 \mathrm{~mm}$ ( $\mathrm{LoE} 2 \mathrm{bB} \mathrm{AGO}+/-)$. Adjuvant trastuzumab plus pertuzumab is recommended in patients with lymph node involvement $(\mathrm{pN}+)(\mathrm{LoE} 1 \mathrm{bB} \mathrm{AGO}+)$ and is an option only in some patients without lymph node involvement $(\mathrm{LoE} 1 \mathrm{bB} A G O+/-)$ but at increased risk [1]. The German experts therefore agree with the majority vote of the SG-BCC panellists (94\%) that patients with HER2 + breast cancer without LN involvement should not receive adjuvant pertuzumab in addition to trastuzumab.

\section{Adjuvant use of neratinib?}

The adjuvant use of neratinib in patients with prior (neo)adjuvant trastuzumab/pertuzumab and/or trastuzumab emtansine (T-DM1) regimen is supported by $63 \%$ of the SG-BCC panellists in the positive ER $(E R+)$ and high risk of recurrence (for example, $\geq 4$ involved $L N)$. The AGO Mamma recommends that patients with $E R+/ H E R 2+$ breast cancer who have already received one year of trastuzumab should continue treatment with neratinib for one year in combination with standard endocrine therapy as an option (LoE $1 \mathrm{bB} \mathrm{AGO+)}$, and in the post-neoadjuvant setting on an individual basis in non-pCR patients (LoE $2 \mathrm{bB} A G O+/-)$ [1]. Due to a lack of data, there is no recommendation on the use of neratinib following trastuzumab/pertuzumab and T-DM1 treatment.

The German experts agree with the majority vote of the SG-BCC panellists, as the AGO Mamma sees a possible indication in ER-positive cases at increased risk. The German experts note the potential adverse events of neratinib, which must be discussed with the patient.

\section{Anthracyclines and anti-HER2 therapy}

A clear majority (76\%) of SG-BCC panellists agree that there are patients with HER2 + breast cancer who can receive an anthracycline sequentially in addition to anti-HER2 therapy combined with anthracycline-free chemotherapy. The German experts agree with this vote. Sequential anthracycline administration is an option depending on individual risk and individual (in particular cardiac) comorbidities. 


\section{T-DM1 instead of trastuzumab/paclitaxel?}

Two-thirds of SG-BCC panellists (69\%) see no indication for T-DM1 in the adjuvant setting in patients with HER2+ stage I breast cancer, while $31 \%$ would consider adjuvant T-DM1 in special circumstances. The German experts agree and add that T-DM1 is not approved for this situation because there are no study data for such an approach.

\section{Adjuvant Systemic Therapy in TNBC}

\section{Adjuvant PD1-/PD-L1-targeted therapy in TNBC?}

About $90 \%$ of SG-BCC panellists see no indication for PD/PD L1targeted immune checkpoint inhibitors in addition to adjuvant chemotherapy in patients with stage II/III TNBC. The German experts agree with the SG-BCC majority vote and note the insufficient data and lack of approval of immune checkpoint inhibitors in early TNBC. In terms of adjuvant treatment, reference is made to the currently ongoing Alexandra trial [10]. They add that patients with early TNBC and chemotherapy indication should primarily receive neoadjuvant treatment [1].

\section{Adjuvant use of PARP inhibition?}

One hopeful therapeutic perspective in early BRCA1/2-associated breast cancer is the use of PARP inhibitors. In terms of the future outcomes of the OlympiA trial [3], 48\% of SG-BCC panellists would support adjuvant olaparib in BRCA1/2-associated breast cancer if after three years of follow-up the OlympiA trial will show an absolute benefit in invasive disease-free survival (iDFS) of $>5 \%$ in the olaparib arm versus the control arm. From the German perspective, we should wait for the presentation of the outcome data at the ASCO meeting in June 2021.

\section{Surgical Issues}

\section{BCS plus radiotherapy in locally recurrent breast cancer}

If a patient is diagnosed with intramammary recurrence and/or ipsilateral second breast cancer more than five years after surgery for the primary tumour followed by radiotherapy, $63 \%$ of the SG-BCC panellists see BCS plus radiotherapy as a suitable alternative to mastectomy. The German experts agree in principle, but still recommend as primary option mastectomy ( $\mathrm{LoE} 3 \mathrm{~b} \mathrm{AGO++}$ ). According to the AGO Mamma, BCS is an option if subsequent (partial) irradiation of breast is possible (LoE 2bB AGO+) [1].

\section{BCS for patients with recurrence?}

The majority of SG-BCC panellists see BCS as an option in patients with intramammary recurrence if it is a low-risk situation (small cancer of the luminal A type) (majority vote: $81 \%$ ) or if the initial diagnosis occurred at least five years previously (majority vote: $64 \%)$. The German experts agree that BCS is justifiable in small cancers and/or luminal A type. Moreover, a time limit cannot be defined. The longer since the initial diagnosis, the less likely is a local recurrence, but rather a second cancer. In everyday clinical practice, however, the distinction is not allways possible.
If repeat radiotherapy is not possible in a patient with intramammary recurrence, the question arises as to whether BCS is appropriate. The SG-BCC vote was 50:50, reflecting the complexity of decision-making. If radiotherapy following BCS is not an option, the German experts recommend the discussion with the patient should primarily focus on mastectomy with possible reconstruction (LoE 3bB AGO++) [1].

\section{No axillary intervention?}

The majority ( $83 \%$ ) of the SG-BCC panellists feel that no axillary intervention is needed in patients over the age of 70 if there are no clinically suspect lymph nodes (cN0). The German experts point out that the decision does not depend solely on the age of the patient, but that comorbidities, the risk of recurrence and possible therapeutic consequences must be considered and discussed with the patient. The decision for or against axillary surgery should only be taken after detailed information. This is also reflected in the recommendations of the AGO Mamma [1]. The German experts also refer to the INSEMA trial [52].

\section{No surgery following NAST?}

With a clear majority (86.00\%), the SG-BCC panellists reject omitting that surgery in patients with early breast cancer and clinical as well as radiological complete response with NAST. The German experts agree with this vote (majority vote: $84 \%$ ).

\section{Surgical approach in the axilla}

The SG-BCC panellists did not agree on the question of whether to remove more than ten $\mathrm{LN}$ in high axillary tumour burden (> $5 \mathrm{LN}$ involved). The German experts point out that ALND is defined in terms of its anatomical boundaries and is not based on the number of removed lymph nodes. The goal is to clear the axilla of all tumour manifestation.

The SG-BCC panellists also failed to reach a consensus on the question of surgical approach in the axilla in patients who, after BCS and in an NO situation ("sentinel node mapping"), currently present with ipsilateral recurrence without $L N$ involvement on imaging. About one third of the SG-BCC panellists recommend SLNE with or without frozen section and $12 \%$ favour ALND, while $20 \%$ are against axillary surgery. The German experts do not agree with the interventions in the axilla. According to AGO Mamma recommendations, SLNE in cN0 patients after primary SLNE (LoE $2 \mathrm{aB}$ AGO-) is not indicated [1].

\section{Oligometastasis}

\section{Curative intent in isolated metastasis}

In patients with clinical stage T2N1 breast cancer and isolated bone metastasis, the SG-BCC panellists (85\%) recommend a curative therapeutic approach with optimal systemic therapy and radiotherapy of the isolated metastasis. The German experts agree with the maximum curative approach whenever there is a chance of cure or long-term survival. 


\section{Intensive treatment even in case of 3-5 metastases}

If a patient (cT2N1) is diagnosed with multiple (>3) bone metastases confirmed by fine-needle biopsy and the tumour is no longer clinically detectable in the breast and axilla after 6 months of systemic therapy with excellent clinical response in the bone, the SG-BCC panellists $(69 \%)$ recommend a palliative concept with regard to local and loco-regional control. The German experts do not agree with the SG-BCC majority vote for a palliative loco-regional concept, but recommend at least considering the continuation of a multimodal approach with curative intent.

\section{Follow-up Care and Quality of Life in Breast Cancer Patients}

\section{Intravaginal oestrogens for mucosal dryness}

If a patient on adjuvant Al therapy experiences mucosal or vaginal dryness that cannot be adequately relieved with moisturisers or lubricants, SG-BCC panellists (73\%) recommend intravaginal oestrogens for symptomatic relief. The German experts agree in principle, but caution that only topical vaginal oestriol containing oestrogens may be used. These are also considered safe for patients with ER+ breast cancer and do not negatively affect treatment success [53]. The issue should be addressed proactively. It is important to maintain compliance with the systemic therapy.

\section{Scalp cooling to prevent alopecia}

SG-BCC panellists (69\%) recommend that patients receiving chemotherapy associated with a risk of alopecia be routinely offered scalp cooling. The German experts agree in principle [54]. This prevents higher-grade alopecia in $40-50 \%$ of patients $[55,56]$. The patients must be informed that side effects, especially headaches, may occur as a result of the strong cooling of the scalp. However, coverage by health insurance is still limited in Germany at present.

\section{Aiming for physical activity}

The SG-BCC panellists particularly recommend physical activity and exercise (44\%) as well as acupuncture (20\%) and normalisation of body weight ( $20 \%$ ) to alleviate disease- and treatment-related secondary symptoms of breast cancer. From the German perspective, all the above proposals are important options and should be pursued according to individual needs and preferences. The best data is on 3-5 hours of physical activity per week (LoE $1 \mathrm{bA} \mathrm{AGO++)}$. Other measures can be found in the recommendations of the AGO Mamma [1].

\section{Reducing alcohol consumption}

Reduced alcohol consumption can help reduce the risk of recurrence in breast cancer patients. The majority of SG-BCC panellists (57\%) recommend a maximum of one alcoholic drink per day. The AGO Mamma recommends limiting daily alcohol consumption to a maximum of $6 \mathrm{~g} /$ day (LoE $2 \mathrm{bB} \mathrm{AGO+}$ ) [1]. Regardless of this recommendation, it is important to set realistic goals. The general rule is that the less alcohol the better.

\section{Comments}

The post-St. Gallen meeting was organised and conducted by Aurikamed Institute $\mathrm{GmbH}$ and made possible by a non-restricted grant from AstraZeneca $\mathrm{GmbH}$, Daiichi Sankyo Deutschland $\mathrm{GmbH}$, Exact Sciences Deutschland GmbH, Lilly Deutschland $\mathrm{GmbH}$, Mylan Germany GmbH, Pierre Fabre Pharma GmbH, Veracyte, Inc. The responsibility for preparing the manuscript rested solely with the authors of the "writing committee". The authors would like to thank Ms. Birgit-Kristin Pohlmann, Nordkirchen, for her editorial support during the preparation of the manuscript.

\section{Conflict of Interest}

Prof. Michael Untch: Honoraria to employer (for AdBoard participation, lectures) and travel support from Amgen; AstraZeneca; BMS; Celgene; Daiichi Sankyo; Eisai; Janssen Cilag; Johnson \& Johnson; Lilly Germany; Lilly International; MSD Merck; Mundipharma; Myriad Genetics; Odonate; Pfizer; PUMA Biotechnology; Riemser; Roche; Pierre Fabre; Novartis; Abbvie; Molecular Health; Agendia; GSK.

Prof. Peter A. Fasching received grants from Biontech, Cepheid and honoraria from Novartis; Pfizer; Daiichi Sankyo; AstraZeneca; Esai; Merck Sharp \& Dohme; Lilly; Pierre Fabre; SeaGen; Roche; Hexal; Agendia. Prof. Sara Y. Brucker; M. D., Ph. D., received honoraria from Roche, Novartis; Pfizer; AstraZeneca; Teva.

Prof. Wilfried Budach, M.D., Ph. D. reports no conflict of interest. Prof. Carsten Denkert, M. D., Ph. D. received honoraria from Novartis; Roche; MSD Oncology; Daiichi Sankyo; Molecular Health; AstraZeneca; Merck; and research funding (to the institution) from Myriad Genetics and Roche. He was co-founder and (until 2016) shareholder of Sividon Diagnostics. He is co-inventor of the VMscope software for Ki-67 analysis.

Renate Haidinger reports no conflict of interest. Prof. Jens Huober received honoraria from Celgene; Roche; Novartis; Hexal; Pfizer; AstraZeneca; Lilly; Amgen; Eisai; MSD; and research support from Celgene, Hexal, Novartis, and travel grants from Roche, Novartis, Daiichi and Celgene.

Prof. Christian Jackisch has received honoraria from AstraZeneca; Lilly; Celgene; Novartis; Pfizer; Roche; and research funding from Exact Sciences.

Prof. Wolfgang Janni received honoraria from Amgen; AstraZeneca; Celgene; Daiichi Sankyo; Eisai; Genomic Health; Hexal; Lilly; MSD; Novartis; Pfizer; Roche; Tesaro; Teva.

Prof. Cornelia Kolberg-Liedtke received honoraria from Roche; AstraZeneca; Celgene; Novartis; Pfizer; Lilly; Hexal; Amgen; Eisai; SonoScape; and consulting fees from Phaon Scientific; Novartis; Pfizer; Celgene; and research funding from Roche, Novartis, Pfizer. Travel Grant from Novartis and Roche and employment at Palleos Healthcare, and Managing Director and shareholder of Phaon Scientific.

David Krug, MD, received honoraria from Merck Sharp \& Dohme. Prof. Thorsten Kühn received honoraria from Celgene; Roche; Pfizer; and research funding from Mammotome, Merit Medical, Pfizer. Prof. Sibylle Loibl, M.D., Ph.D., received honoraria from AbbVie, Amgen, AstraZeneca, Bayer, BMS, Celgene, Chugai, Daiichi Sankyo, Eirgenix, GSK, Immunomedics, Ipsen, Lilly, Merck, Novartis, Pfizer, Pierre Fabre, Prime/Medscape, Puma, Roche, Samsung, Seagen, Teva, Vifor, and has a patent application EP14153692.0 pending.

Prof. Diana Lüftner received honoraria from Amgen; AstraZeneca; Celgene; Pfizer; Novartis; Amgen; Roche; Loreal; Teva; GSK; and Eli Lilly. Prof. Volkmar Müller received honoraria from Amgen; AstraZeneca; Daiichi Sankyo; Eisai; Pfizer; MSD; Novartis; Roche; Teva; Seattle Genetics; and for consulting services from Genomic Health; Hexal; Roche; Pierre Fabre; Amgen; ClinSol; Novartis; MSD; Daiichi Sankyo; Eisai; Lilly; Tesaro; Seattle Genetics; Neltar; and travel expenses from 
Roche; Pfizer; Daiichi Sankyo; and research funding to the employer from Novartis; Roche; Seattle Genetics; Genentech. Prof. Andreas Schneeweiss received honoraria from Celgene; Roche; Pfizer; AstraZeneca; Novartis; MSD; Tesaro; Lilly; and research funding from Celgene; Roche; AbbVie; Molecular Partner; and travel grants from Celgene, Roche and Pfizer.

Prof. Dr. Marc Thill received honoraria and travel support from Amgen; AstraZeneca; Aurikamed; Becton and Dickinson, Biom'Up; Celgene; Clearcut; Connect Medica; Daiichi Sankyo; Eisai; Exact Sciences; Grünenthal; Hexal; I-Med Institute; Lilly; MCI; Medtronic; MSD; Neodynamics; Norgine; Novartis; OnkoLive; OmniaMed, Organon, pfm Medical; Pfizer; Roche; Seagen; Sysmex; Tesaro; Teva; RTI Surgical; Vifor; and research funding from Endomagnetics and Exact Sciences. Prof. Nadia Harbeck received honoraria from AstraZeneca; Daiichi Sankyo; Lilly; MSD; Novartis; Pierre Fabre; Pfizer; Roche; Seattle Genetics.

Prof. Christoph Thomssen received honoraria from Amgen; AstraZeneca; Celgene; Daiichi Sankyo; Eisai; Lilly; MSD; Mylan; Nanostring; Novartis; Pfizer; Pierre Fabre; Puma; Roche; Vifor; and research funding from American Diagnostica, Affymetrix, Nanostring.

\section{References}

[1] AGO Kommission Mamma. Diagnostik und Therapie früher und fortgeschrittener Mammakarzinome (März 2021). Accessed April 18, 2021 at: https://www.ago-online.de/fileadmin/ago-online/downloads/ _leitlinien/kommission_mamma/2021/Alle_aktuellen_Empfehlungen_ 2021.pdf

[2] Interdisziplinäre S3-Leitlinie für die Früherkennung, Diagnostik, Therapie und Nachsorge des Mammakarzinoms [Langversion 4.3 (Februar 2020). Accessed April 18, 2021 at: https://www.awmf.org/uploads/tx_ szleitlinien/032-045OLI_S3_Mammakarzinom_2020-02.pdf

[3] Breast International Group. Olaparib as Adjuvant Treatment in Patients With Germline BRCA Mutated High Risk HER2 Negative Primary Breast Cancer (OlympiA). Accessed April 18, 2021 at: https://clinicaltrials.gov/ ct2/show/NCT02032823

[4] Nielsen TO, Leung SCY, Rimm DL et al. Assessment of Ki67 in Breast Cancer: Updated Recommendations from the International Ki67 in Breast Cancer Working Group. J Natl Cancer Inst 2020. doi:10.1093/jnci/ djaa201

[5] Fasching PA, Gass P, Häberle L et al. Prognostic effect of Ki-67 in common clinical subgroups of patients with HER2-negative, hormone receptor-positive early breast cancer. Breast Cancer Res Treat 2019; 175: 617625. doi:10.1007/s10549-019-05198-9

[6] Wiesner FG, Magener A, Fasching PA et al. Ki-67 as a prognostic molecular marker in routine clinical use in breast cancer patients. Breast 2009; 18: 135-141. doi:10.1016/j.breast.2009.02.009

[7] Dettmar P, Harbeck N, Thomssen C et al. Prognostic impact of proliferation-associated factors MIB1 (Ki-67) and S-phase in node-negative breast cancer. $\mathrm{Br}$ J Cancer 1997; 75: 1525-1533. doi:10.1038/ bjc. 1997.261

[8] Harbeck N, Gluz O, Kuemmel S et al. Neoadjuvant nab-paclitaxel weekly versus dose-dense paclitaxel followed by dose-dense $\mathrm{EC}$ in high risk HR +/HER2- early BC by: Results from the neoadjuvant part of ADAPT HR +/HER2- trial. San Antonio Breast Cancer Symposium 2020; Abstract No. GS4-03

[9] Smith I, Robertson J, Kilburn L et al. Long-term outcome and prognostic value of Ki67 after perioperative endocrine therapy in postmenopausal women with hormone-sensitive early breast cancer (POETIC): an openlabel, multicentre, parallel-group, randomised, phase 3 trial. Lancet Oncol 2020; 21: 1443-1454. doi:10.1016/S1470-2045(20)30458-7

[10] German Breast Group. A Study Comparing Atezolizumab (Anti PD-L1 Antibody) In Combination With Adjuvant Anthracycline/Taxane-Based Chemotherapy Versus Chemotherapy Alone In Patients With Operable
Triple-Negative Breast Cancer (IMpassion030). Accessed April 18, 2021 at: https://www.clinicaltrials.gov/ct2/show/NCT03498716

[11] NSABP Foundation Inc.; German Breast Group. Clinical Trial of Neoadjuvant Chemotherapy With Atezolizumab or Placebo in Patients With Triple-Negative Breast Cancer Followed After Surgery by Atezolizumab or Placebo - GeparDouze (GBG 96). Accessed April 18, 2021 at: https:// clinicaltrials.gov/ct2/show/NCT03281954

[12] Study to Compare a Mono Atezolizumab Window Followed by a Atezolizumab - CTX Therapy With Atezolizumab - CTX Therapy (neoMono). Accessed April 18, 2021 at: www.clinicaltrials.gov/ct2/show/ NCT04770272?term=neomono\&draw $=2 \&$ rank $=1$

[13] European Breast Cancer Reseach Association of Surgical Trialists. AXillary Surgery After NeoAdjuvant Treatment (AXSANA). Accessed April 18, 2021 at: https://clinicaltrials.gov/ct2/show/NCT04373655

[14] Kuemmel S, Heil J, Rueland A et al. A Prospective, Multicenter Registry Study to Evaluate the Clinical Feasibility of Targeted Axillary Dissection (TAD) in Node-Positive Breast Cancer Patients. Ann Surg 2020. doi:10.1097/SLA.0000000000004572

[15] Caudle AS, Yang WT, Krishnamurthy $S$ et al. Improved Axillary Evaluation Following Neoadjuvant Therapy for Patients With Node-Positive Breast Cancer Using Selective Evaluation of Clipped Nodes: Implementation of Targeted Axillary Dissection. J Clin Oncol 2016; 34: 1072-1078. doi:10.1200/JCO.2015.64.0094

[16] Giuliano AE, Ballman KV, McCall L et al. Effect of Axillary Dissection vs. No Axillary Dissection on 10-Year Overall Survival Among Women With Invasive Breast Cancer and Sentinel Node Metastasis: The ACOSOG Z0011 (Alliance) Randomized Clinical Trial. JAMA 2017; 318: 918-926. doi:10.1001/jama.2017.11470

[17] Donker M, van Tienhoven G, Straver ME et al. Radiotherapy or surgery of the axilla after a positive sentinel node in breast cancer (EORTC 1098122023 AMAROS): a randomised, multicentre, open-label, phase 3 noninferiority trial. Lancet Oncol 2014; 15: 1303-1310. doi:10.1016/ S1470-2045(14)70460-7

[18] NSABP Foundation Inc. Standard or Comprehensive Radiation Therapy in Treating Patients With Early-Stage Breast Cancer Previously Treated With Chemotherapy and Surgery. Accessed April 18, 2021 at: https:// clinicaltrials.gov/ct2/show/study/NCT01872975

[19] Brunt AM, Haviland J, Sydenham M et al. FAST Phase III RCT of Radiotherapy Hypofractionation for Treatment of Early Breast Cancer: 10-Year Results (CRUKE/04/015). International Journal of Radiation Oncology*Biology*Physics 2018; 102: 1603-1604. doi:10.1016/j.jjrobp.2018.08.049

[20] Murray Brunt A, Haviland JS, Wheatley DA et al. Hypofractionated breast radiotherapy for 1 week versus 3 weeks (FAST-Forward): 5 -year efficacy and late normal tissue effects results from a multicentre, non-inferiority, randomised, phase 3 trial. The Lancet 2020; 395: 1613-1626. doi:10.1016/S0140-6736(20)30932-6

[21] Krug D, Baumann R, Combs SE et al. Moderate hypofractionation remains the standard of care for whole-breast radiotherapy in breast cancer: Considerations regarding FAST and FAST-Forward. Strahlenther Onkol 2021; 197: 269-280. doi:10.1007/s00066-020-01744-3

[22] Strnad V, Krug D, Sedlmayer $F$ et al. DEGRO practical guideline for partial-breast irradiation. Strahlenther Onkol 2020; 196: 749-763. doi:10.1007/s00066-020-01613-z

[23] Evron E, Ben-David AM, Goldberg $\mathrm{H}$ et al. Prophylactic irradiation to the contralateral breast for BRCA mutation carriers with early-stage breast cancer. Ann Oncol 2019; 30: 412-417. doi:10.1093/annonc/mdy515

[24] Krug D, Baumann R, Budach W et al. Commercially Available Gene Expression Assays as Predictive Tools for Adjuvant Radiotherapy? A Critical Review. Breast Care (Basel) 2020; 15: 118-126. doi:10.1159/000505656

[25] van der Noordaa ME, Yau C, Shad S et al. Assessing prognosis after neoadjuvant therapy: A comparison between anatomic ypAJCC staging, Residual Cancer Burden Class and Neo-Bioscore. San Antonio Breast Cancer Symposium 2020; Abstract No. GS4-07 
[26] Mittendorf EA, Vila J, Tucker SL et al. The Neo-Bioscore Update for Staging Breast Cancer Treated With Neoadjuvant Chemotherapy: Incorporation of Prognostic Biologic Factors Into Staging After Treatment. JAMA Oncol 2016; 2: 929-936. doi:10.1001/jamaoncol.2015.6478

[27] Jeruss JS, Mittendorf EA, Tucker SL et al. Combined use of clinical and pathologic staging variables to define outcomes for breast cancer patients treated with neoadjuvant therapy. J Clin Oncol 2008; 26: 246252. doi:10.1200/JCO.2007.11.5352

[28] von Minckwitz G, Huang C-S, Mano MS et al. Trastuzumab Emtansine for Residual Invasive HER2-Positive Breast Cancer. N Engl J Med 2019; 380: 617-628. doi:10.1056/NEJMoa1814017

[29] German Breast Group. Sacituzumab Govitecan in Primary HER2-negative Breast Cancer (SASCIA). Accessed April 18, 2021 at: https://clinicaltrials. gov/ct2/show/NCT04595565

[30] McCormick B, Winter K, Hudis C et al. RTOG 9804: a prospective randomized trial for good-risk ductal carcinoma in situ comparing radiotherapy with observation. J Clin Oncol 2015; 33: 709-715. doi:10.1200/JCO.2014.57.9029

[31] Chua BH, Link E, Kunkler I et al. GS2-04. A randomized phase III study of radiation doses and fractionation schedules in non-low risk ductal carcinoma in situ (DCIS) of the breast (BIG 3-07/TROG 07.01). San Antonio Breast Cancer Symposium 2020; Abstract No. GS2-04

[32] Wang S-L, Fang H, Song Y-W et al. Hypofractionated versus conventional fractionated postmastectomy radiotherapy for patients with high-risk breast cancer: a randomised, non-inferiority, open-label, phase 3 trial. Lancet Oncol 2019; 20: 352-360. doi:10.1016/S1470-2045(18)30813-1

[33] Danish Breast Cancer Cooperative Group. Hypofractionated Loco-regional Adjuvant Radiation Therapy of Breast Cancer Combined With a Simultaneous Integrated Boost. Accessed April 18, 2021 at: https:// clinicaltrials.gov/ct2/show/NCT02384733

[34] Alliance for Clinical Trials in Oncology. Hypofractionated Radiation Therapy After Mastectomy in Preventing Recurrence in Patients With Stage II a-III a Breast Cancer. Accessed April 18, 2021 at: https://clinicaltrials. gov/ct2/show/NCT03414970

[35] Dana-Farber Cancer Institute. Study of Radiation Fractionation on Patient Outcomes After Breast REConstruction (FABREC) for Invasive Breast Carcinoma. Accessed April 18, 2021 at: https://clinicaltrials.gov/ct2/ show/NCT03422003

[36] M.D. Anderson Cancer Center. Hypofractionated vs. Conventional Regional Nodal Radiation Therapy for Patients With Invasive Breast Cancer. Accessed April 18, 2021 at: https:/clinicaltrials.gov/ct2/show/ NCT02912312

[37] Marta GN, Ramiah D, Kaidar-Person O et al. The Financial Impact on Reimbursement of Moderately Hypofractionated Postoperative Radiation Therapy for Breast Cancer: An International Consortium Report. Clin Oncol (R Coll Radiol) 2020. doi:10.1016/j.clon.2020.12.008

[38] Krug D, Vonthein R, Schreiber A et al. Impact of guideline changes on adoption of hypofractionation and breast cancer patient characteristics in the randomized controlled HYPOSIB trial. Strahlenther Onkol 2020. doi:10.1007/s00066-020-01730-9

[39] Kunkler I, Williams LJ, Jack W et al. GS2-03. Prime 2 randomised trial (postoperative radiotherapy in minimum-risk elderly): Wide local excision and adjuvant hormonal therapy $+/$ - whole breast irradiation in women $=\mid>65$ years with early invasive breast cancer: 10 year results. San Antonio Breast Cancer Symposium 2020; Abstract No. GS2-03

[40] Vaidya JS, Wenz F, Bulsara M et al. Risk-adapted targeted intraoperative radiotherapy versus whole-breast radiotherapy for breast cancer: 5-year results for local control and overall survival from the TARGIT-A randomised trial. Lancet 2014; 383: 603-613. doi:10.1016/S0140-6736(13) 61950-9

[41] Vaidya JS, Bulsara M, Baum M et al. Long term survival and local control outcomes from single dose targeted intraoperative radiotherapy during lumpectomy (TARGIT-IORT) for early breast cancer: TARGIT-A randomised clinical trial. BMJ 2020; 370: m2836. doi:10.1136/bmj.m2836
[42] O'Shaughnessy JA, Johnston S, Harbeck N et al. Primary outcome analysis of invasive disease-free survival for monarchE: abemaciclib combined with adjuvant endocrine therapy for high risk early breast cancer. San Antonio Breast Cancer Symposium 2020; Abstract No. GS1-01

[43] Cardoso F, van't Veer L], Bogaerts ] et al. 70-Gene Signature as an Aid to Treatment Decisions in Early-Stage Breast Cancer. N Engl J Med 2016; 375: 717-729. doi:10.1056/NEJMoa1602253

[44] Sparano JA, Gray RJ, Della Makower F et al. Prospective Validation of a 21-Gene Expression Assay in Breast Cancer. N Engl J Med 2015; 373 : 2005-2014. doi:10.1056/NEJMoa1510764

[45] Kalinsky K, Barlow WE, Meric-Bernstam F et al. First results from a phase III randomized clinical trial of standard adjuvant endocrine therapy (ET) +/- chemotherapy (CT) in patients (pts) with 1-3 positive nodes, hormone receptor-positive (HR+) and HER2-negative (HER2-) breast cancer (BC) with recurrence score (RS) <25: SWOG S1007 (RxPonder). San Antonio Breast Cancer Symposium 2020; Abstract No. GS3-00

[46] West German Study Group. Adj. Dyn. Marker-adjusted Personalized Therapy Comparing Abemaciclib + SOC ET vs. SOC ET in Clinical or Genomic High Risk, HR+/HER2- EBC (ADAPTlate). Accessed April 18 2021 at: https://clinicaltrials.gov/ct2/show/NCT04565054

[47] West German Study Group. Adj. Marker-adjusted Personalized Therapy Comparing ET+Ribociclib vs. Chemotherapy in Intermediate Risk, HR+/HER2- EBC (ADAPTcycle). Accessed April 18, 2021 at: https:// clinicaltrials.gov/ct2/show/NCT04055493

[48] Gonzalez-Angulo AM, Litton JK, Broglio KR et al. High risk of recurrence for patients with breast cancer who have human epidermal growth factor receptor 2-positive, node-negative tumors $1 \mathrm{~cm}$ or smaller. J Clin Oncol 2009; 27: 5700-5706. doi:10.1200/JCO.2009.23.2025

[49] Kang Y-J, Oh S], Choi H et al. Clinical significance of HER2 status in T1 bN0 breast cancer: a nationwide study from the Korean Breast Cancer Society. Breast Cancer Res Treat 2021; 186: 125-134. doi:10.1007| s10549-020-06017-2

[50] Cancello G, Maisonneuve P, Rotmensz N et al. Prognosis in women with small (T1mic,T1a,T1b) node-negative operable breast cancer by immunohistochemically selected subtypes. Breast Cancer Res Treat 2011; 127: 713-720. doi:10.1007/s10549-011-1465-7

[51] Villasco A, Actis S, Borella F et al. Evaluation of the efficacy of Trastuzumab in patients with HER2 positive small (pT1mi/a) breast cancers. A multicenter retrospective study on 100 patients. The Breast 2021; 56 : S17-S18. doi:10.1016/S0960-9776(21)00090-4

[52] Reimer T, Stachs A, Nekljudova V et al. Restricted Axillary Staging in Clinically and Sonographically Node-Negative Early Invasive Breast Cancer (c/iT1-2) in the Context of Breast Conserving Therapy: First Results Following Commencement of the Intergroup-Sentinel-Mamma (INSEMA) Trial. Geburtshilfe Frauenheilkd 2017; 77: 149-157. doi:10.1055/ s-0042-122853

[53] Cold S, Cold F, Jensen M-B et al. 1814 Vaginal estrogens and risk of recurrence or death in women treated for estrogen receptor positive breast cancer. Eur J Cancer 2015; 51: S270. doi:10.1016/S0959-8049 (16)30766-3

[54] Smetanay K, Junio P, Feißt M et al. COOLHAIR: a prospective randomized trial to investigate the efficacy and tolerability of scalp cooling in patients undergoing (neo)adjuvant chemotherapy for early breast cancer. Breast Cancer Res Treat 2019; 173: 135-143. doi:10.1007/s10549-0184983-8

[55] Rugo HS, Voigt J. Scalp Hypothermia for Preventing Alopecia During Chemotherapy. A Systematic Review and Meta-Analysis of Randomized Controlled Trials. Clin Breast Cancer 2018; 18: 19-28. doi:10.1016/j. clbc.2017.07.012

[56] Nangia J, Wang T, Osborne C et al. Effect of a Scalp Cooling Device on Alopecia in Women Undergoing Chemotherapy for Breast Cancer: The SCALP Randomized Clinical Trial. JAMA 2017; 317: 596-605. doi:10.1001/jama.2016.20939 